Provided for non-commercial research and education use. Not for reproduction, distribution or commercial use.

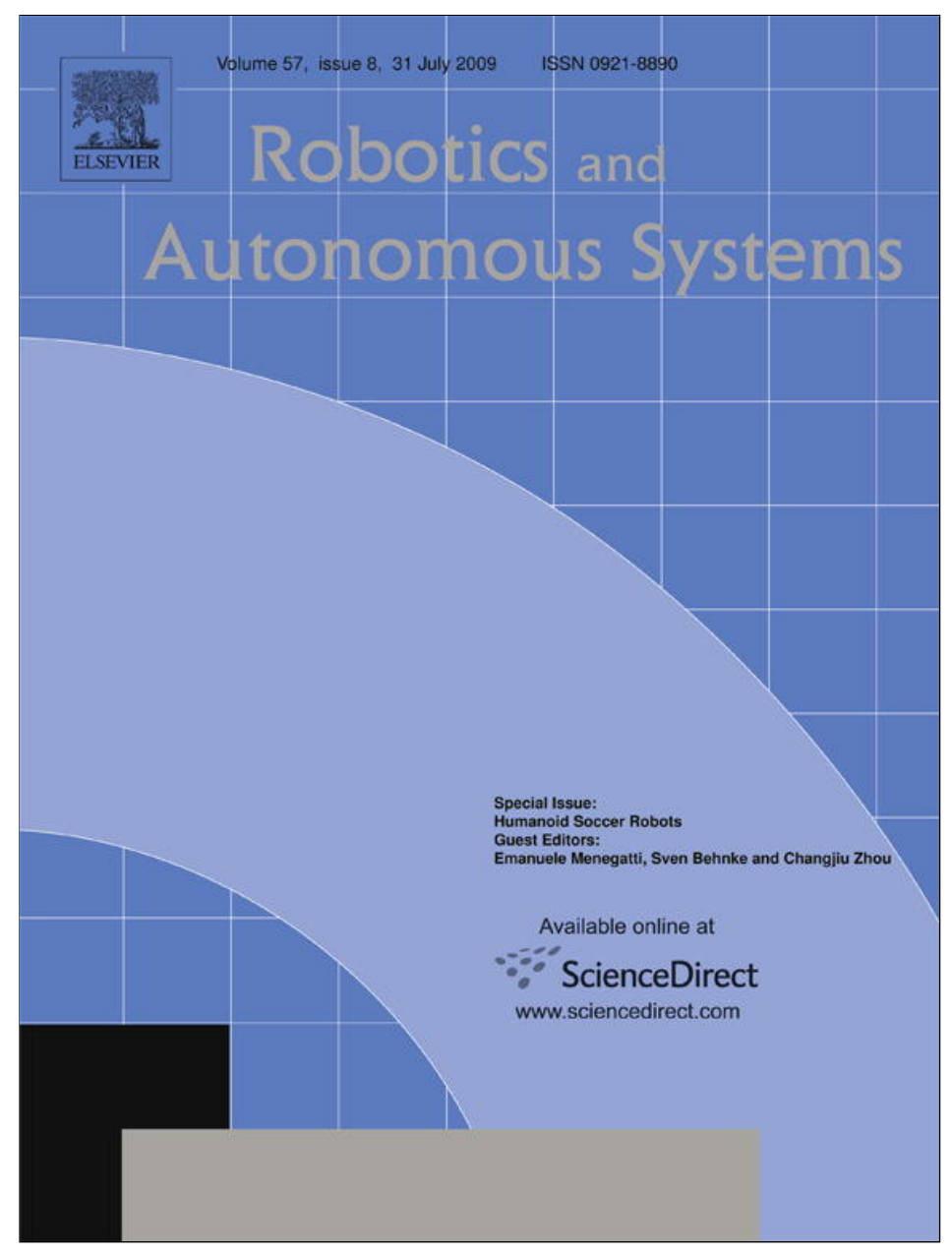

This article appeared in a journal published by Elsevier. The attached copy is furnished to the author for internal non-commercial research and education use, including for instruction at the authors institution and sharing with colleagues.

Other uses, including reproduction and distribution, or selling or licensing copies, or posting to personal, institutional or third party websites are prohibited.

In most cases authors are permitted to post their version of the article (e.g. in Word or Tex form) to their personal website or institutional repository. Authors requiring further information regarding Elsevier's archiving and manuscript policies are encouraged to visit:

http://www.elsevier.com/copyright 


\title{
Kick it with elasticity: Safety and performance in human-robot soccer
}

\author{
Sami Haddadin ${ }^{\mathrm{a}, *}$, Tim Laue $^{\mathrm{b}}$, Udo Frese ${ }^{\mathrm{b}}$, Sebastian Wolf ${ }^{\mathrm{a}}$, Alin Albu-Schäffer ${ }^{\mathrm{a}}$, Gerd Hirzinger $^{\mathrm{a}}$ \\ a Institute of Robotics and Mechatronics, DLR - German Aerospace Center, Wessling, Germany \\ ${ }^{\mathrm{b}}$ Safe and Secure Cognitive Systems, DFKI, Enrique-Schmidt-Str. 5, Bremen, Germany
}

\section{A R T I C L E I N F O}

\section{Article history:}

Available online 5 April 2009

\section{Keywords:}

RoboCup

Human-robot soccer

Safe robot

Kicking

Elastic joints

Variable stiffness actuation

\section{Introduction}

\subsection{The RoboCup 2050 challenge}

Soon after establishing the RoboCup competition in 1997, the RoboCup Federation proclaimed an ambitious long-term goal (Fig. 1).

"By mid-21st century, a team of fully autonomous humanoid robot soccer players shall win the soccer game, comply with the official rule of the FIFA, against the winner of the most recent World Cup."

\section{H. Kitano and M. Asada [1]}

Soccer is a contact sport and injuries of players are frequent [2]. Even more, the FIFA rules state explicitly, that

"Football is a competitive sport and physical contact between players is a normal and acceptable part of the game. [... ]"

Laws of the game, 2006 [3]

For a soccer match between humans and robots this implies physical human-robot interaction (pHRI) including tackles and fouls between humans and robots. In order to come closer to

\footnotetext{
* Corresponding author.

E-mail address: sami.haddadin@dlr.de (S. Haddadin).
}

that vision, an evaluation of the fundamental requirements and challenges the human presence would bring into such a match is, in our opinion, absolutely crucial and definitely still an open issue. This makes not only sense from the perspective of ensuring human safety but as well of defining requirements a robot has to fulfill in order to withstand the enormous strains posed by such a real soccer game. These problems can only be approached and tackled if one sees the robotic and biomechanical aspects as complementary.

The first contribution of this paper is to shed light on the pHRI aspects of such a hypothetical human-robot match. Therefore, we use two matches from the recent (2006) FIFA World Cup in Germany as examples and analyze them with respect to scenes with physical interaction. We relate these interactions to results in pHRI and sports science by imagining what would have happened if one of the opponents was a robot.

In the domains of industrial assistance and service robotics, robots are and will be designed to cause absolutely no harm to any human. Presumably, such a robot could never win. However, we demand that a human-robot match should not be more dangerous than an ordinary soccer match. Hence, we focus on situations, where a robot is expected to potentially cause more injury than a human player.

\subsection{Organization of the paper}

After giving a state of the art in physical Human-Robot Interaction (pHRI) in Section 2, PART I of this paper is concerned 


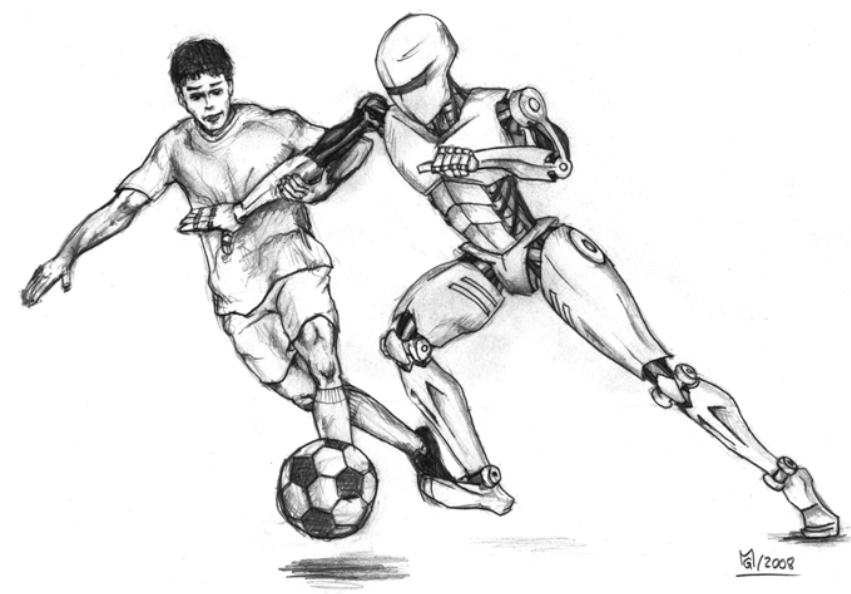

Fig. 1. The RoboCup 2050 challenge.

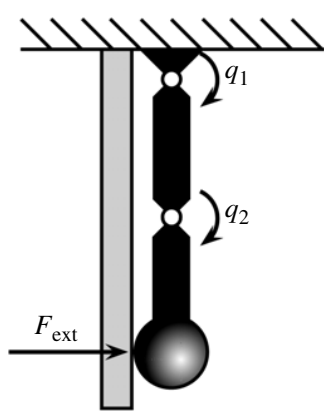

Fig. 2. Example for a robot impact: A two-joint robot with joint angles $q_{1}=q_{2}=0$ deg hits a wall with an extra mass at the TCP. The robot is decelerated by the contact force $F_{\text {ext }}$ imposed by the wall.

with how a robot might hurt a human player during typical fouls in soccer (Section 3). We first classify human soccer fouls and discuss them from a pHRI perspective. Then, we present a simulation and experimental analysis of impacts, in particular elbow checks as a major injury source. PART II focuses on the robot. How can it withstand the impact of kicking the ball or even fouls (Section 4)? And finally, PART III discusses how joint elasticity can be used to achieve the kick velocity of human soccer players (Section 5). The discussion includes experiments with traditional robots with little elasticity, experiments using a joint with large elasticity, and finally a theorem on optimal control of an elastic joint. Table 2 gives a list of symbols.

\section{State of the art in physical Human-Robot Interaction}

\subsection{The dynamics of a robot during impact}

Most of this paper is concerned with situations involving impacts, either with the ball during kicking or with the opponent during tackling. This is rather uncommon for the computer science literature, so we start by describing intuitively what happens during an impact of a two-joint robot arm or leg (Fig. 2).

When an object hits, for example, a wall, the wall imposes a high force on the object decelerating it or even reflecting it back. This happens during a very short time, the impact. For idealized rigid objects, the force is infinity, acting just in a moment. But even for real rigid objects, the duration is in the order of milliseconds and the force of kilo-Newtons. A colliding robot "appears" to the wall as an object approaching with the Cartesian velocity of the robot at the contact point. But what is the "apparent mass", the so-called reflected inertia [4] of the robot? As intuition already suggests, not the full robot mass, because the wall acts with a lever on the mass located in the links. Hence this mass is more easily decelerated and appears lighter to the contact point. Furthermore, the second joint decouples the first link from the second. The forces of a rigid impact are usually vastly higher than actuator forces/torques, so for the impact duration joints can be treated as unactuated. Hence in an outstretched configuration $\left(q_{2}=0 \mathrm{deg}\right)$, the Tool Center Point (TCP) is stopped or reflected back resulting in a "step" in $\dot{q}_{2}$ while the first link simply continues moving. In general however, some of the inertia of the first link appears at the contact point dependent on the angle. The mathematical derivation of the reflected inertia of a manipulator (which will be used in the impact evaluation) is well established in the robotics literature [4].

There is another effect, not so well known. For a rigid joint robot, a step in $\dot{q}_{2}$ results in an impact on the motor inertia. The resulting reflected motor inertia adds to the reflected link inertia at the contact point and can be quite significant for robots with high gear ratio. However, when the joint has an elasticity higher than the elasticity at the contact point, the motor continues moving for some time after the impact. This leads to increasing tension in the joint elasticity which starts decelerating the motor. The resulting effect is a decoupling of motor and link inertia during the impact. Thus, the motor inertia does not contribute to the reflected inertia at the moment of impact. Surprisingly, this happens already for a joint elasticity of typical light-weight robots without any extra elasticity added [5].

Overall, in the example in Fig. 2 and under the assumption of such flexible joints, the reflected inertia consists of the full mass at the TCP and some fraction of the second link. The first link and both motor inertias do not contribute.

\subsection{Design and control for physical Human-Robot interaction}

Recently, there is increasing interest in domestic and industrial service robots that allow physical interaction [6-8]. The goal of robots and humans coexisting in the same physical domain poses various fundamental problems for the entire robotic design. Unlike their classical counterparts, these robots take into account for the hardware design, control and planning that the environment is partially unknown. Such a robot cannot simply move along computed trajectories but must react meaningfully, i.e. compliantly, to unexpected contact with the environment. Therefore, it is usually equipped with proprioceptive sensors, such as Cartesian force/torque and joint torque sensors $[9,10]$ and/or arrays resembling a sensitive skin (especially for hands [11]). Alternatively, backdrivable motors are used to passively react to external forces [12].

The most widely used control approach to physically interact with robots is probably impedance control and its related schemes, introduced in the pioneering work of Neville Hogan in [13] and extended to many classes of robots. This type of controller imposes a desired physical behavior with respect to external forces on the robot. For instance the robot is controlled to behave like a second order mass-spring-damper system. Consequently, impedance control allows us to realize compliance of the robot by means of control.

Interaction with an impedance controlled robot is very robust and intuitive, since in addition to the commanded trajectory, a disturbance response is defined. A major advantage of impedance control (with impedance causality) is that discontinuities like contact-non-contact do not create such stability problems as for example with hybrid force control [14]. However, many open questions still have to be tackled from a control point of view, such as how to adjust the impedance according to the current task. 


\subsection{Introducing joint elasticity into the mechanical design}

Apart from such control issues, mechanical design plays a fundamental role in safety, bringing humans and robots spatially closer. Joint elasticity has long been addressed in light-weight robot construction, however more as an undesired consequence which the control has to handle $[9,15]$. An interesting and promising paradigm currently re-arising in robotics design is antagonism [16,17], or more generally variable stiffness/impedance actuation (VSA/VIA). The idea is to implement joint compliance not by means of control but via adjustable intrinsically compliant joints, inspired by the unquestionably successful design of human and animal muscles. The design and control of such systems were addressed in numerous publications [18-20,17,21,22]. Our paper follows this general line in clearly deriving why elastic joints are necessary for human-robot soccer.

\subsection{Compliance for walking and running}

In this paper we focus on the benefit of elastic joints for safety and kicking performance. Nevertheless, in a soccer scenario this would imply also to walk and run with these joints. So we briefly review the state of the art in this field.

Current large and medium scale anthropometric humanoids as H6, H7 [23], P2 [24], ASIMO [25], JOHNNIE, LOLA [26], WABIAN-2 [27], KHR-2 [28], HRP, HRP-2 [29], and SAIKA [30] represent major achievements over the last years. In these systems, locomotion is mostly realized with stiff actuation in combination with rigid high geared transmission mechanisms. Due to the lack of an appropriate storage mechanism, the entire energy is lost during walking and running and has to be continuously injected by active actuation. The same holds for the robots in the RoboCup domain, where usually no deliberately introduced compliance is used.

However, there exist already some realizations which successfully used intrinsically compliant joint designs for biped walking. In WL-14 [31,32], a sophisticated nonlinear spring mechanism was used for stiffness adjustment. More recently in Lucy [17], a biped that is able to walk in the sagittal plane, approaches were made to utilize adjustable passive compliance for high energy efficiency during walking. The robot Flame [33] uses constant compliance (Series Elastic Actuation) in the hip, knee, and ankle pitch joint. HRP-2LR [34] is equipped with a compliant toe in both feet having a constant rotational spring. The authors predicted via simulation a running speed of $3 \mathrm{~km} / \mathrm{h}$ with this device compared to $0.58 \mathrm{~km} / \mathrm{h}$ achieved with HRP-2LT that has no such compliant toes. Up until now, the authors already demonstrated hopping with both feet.

Apart from these first realizations in the field of biped walking, there is clear evidence in biomechanics that intrinsically compliant actuation is fundamental to terrestrial locomotion [35]. So to summarize, running with elastic joints seems to be difficult but possible and probably of long-term benefit.

\subsection{Safety in physical Human-Robot interaction}

As Asimov already noted very early, safety has priority if robots are close to humans [36]. Fundamental work on human-robot impacts under worst-case conditions and resulting injuries was carried out in $[19,37,5]$, with moderate robot speed up to $2 \mathrm{~m} / \mathrm{s}$. During such unexpected collisions, various injury sources exist: fast impacts, clamping, slow quasi-static loading, or sharp tools. Current results indicate that a robot, even with arbitrary mass, driving up to $2 \mathrm{~m} / \mathrm{s}$ is not able to become dangerous with respect to typical severity measures used in automobile industry, except when clamping the victim $[38,39]$. This does not rule out other injuries, such as linear fractures, cuts, or laceration, but it indicates that, if clamping is prevented, typical physical human-robot
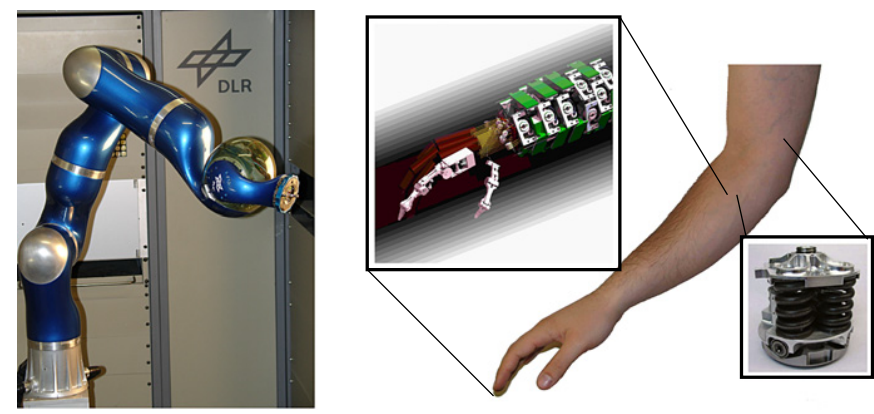

Fig. 3. The DLR LWRIII (left) and the new DLR hand-arm system which anthropomorphic design is aiming at similar capabilities to a human arm (right).

interaction is much less dangerous than indicated in earlier work as [19,37]. ${ }^{1}$

\subsection{A simulation model for a humanoid soccer robot leg}

Simulated and real experiments in this paper primarily refer to the DLR light-weight Robot III (DLR-LWRIII) [9,15], a light-weight robot with some joint elasticity weighing $13 \mathrm{~kg}$ (Fig. 3, left) and the DLR VS-Joint, a prototype developed for the new intrinsically compliant DLR hand-arm system [40,41] (Fig. 3, right). This joint is is a representative of such intrinsically compliant devices and all major conclusions made in this paper related to joint elasticity are of general character.

The DLR-LWRIII is equipped with joint torque sensors and impedance control as necessary for physical interaction. Although being designed as an arm, it has inertial and geometric properties comparable to a human leg ( $\left.\frac{D L R-L W R I I I}{L e g} \approx 1.2\right)[42,43]$. Thus, we use it as a "model" for the leg of a future humanoid soccer robot throughout, while not claiming that the design is feasible for a leg in general. With $130 \mathrm{deg} / \mathrm{s}$ its maximum joint velocity, however, is much lower than that of a human soccer player having $1375 \mathrm{deg} / \mathrm{s}$ [44]. Hence in simulations we often consider a hypothetical, faster DLR-LWRIII as a model.

\section{PART I: Safety of the human}

This part is concerned with typical physical interactions in soccer. After a short overview of collisions in robot soccer, it majorly covers fouls in human soccer. These are classified into different categories and discussed from a pHRI perspective. Afterwards, we present a simulation and experimental analysis of impacts, in particular elbow checks as a major injury source.

\subsection{Physical interaction in humanoid robot soccer}

Most RoboCup Soccer leagues, including the Humanoid league, already base their rules roughly on the official FIFA laws of the game. Thus, physical interaction and fouls are specified together with the resulting consequences [45]. However, the level of detail is much lower than that in the original rules, which even include Additional Instructions and Guidelines for Referees [3] to distinguish different kinds of physical interactions explicitly.

\footnotetext{
1 A correction of the initial misinterpretation in units done in [19] and [37] was first carried out in [69,5]. Instead of using the appropriate units [g] for acceleration $\left[\mathrm{m} / \mathrm{s}^{2}\right]$ was applied in the former work. This leads to an overestimation of HIC in the range of $9.81^{2.5} \approx 301$. Apart from this, some oversimplification of the HIC formula were done which could lead to an error of $\approx 2$. However, the HIC was then corrected as well in $[71,70]$.
} 

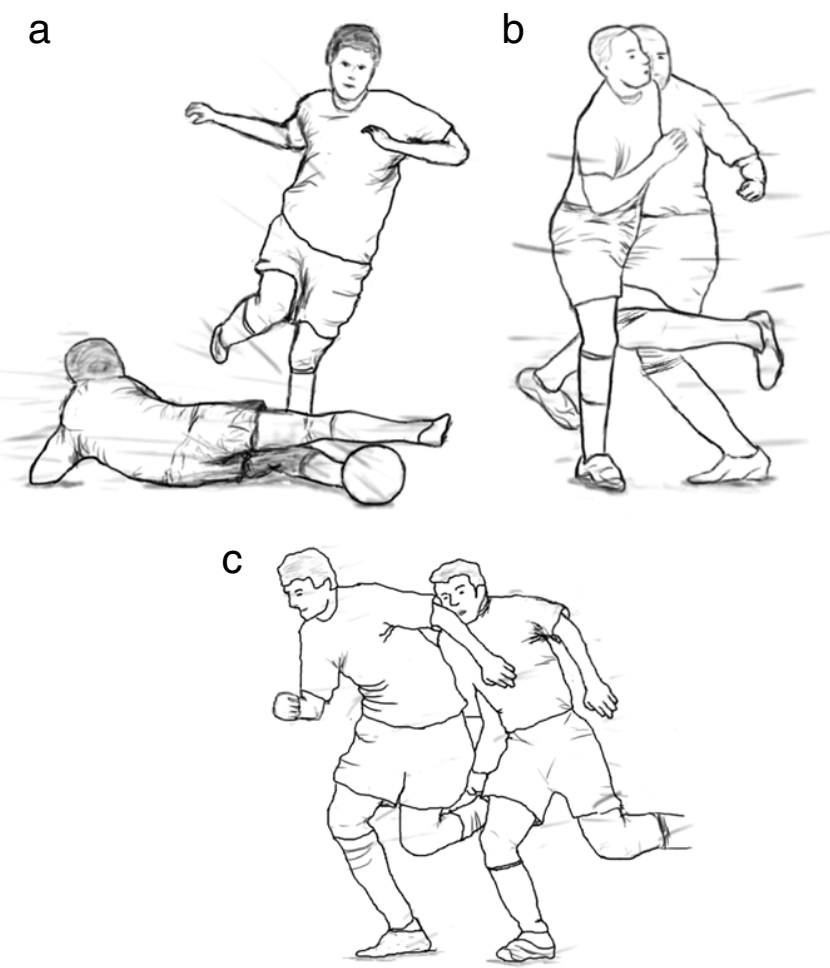

Fig. 4. Scenes from the FIFA world championship 2006 showing different classes of physical interaction. a) tripping b) trunk impacts c) limb impacts (here with the elbow).

Even when having 20 degrees of freedom, current humanoid soccer robots are not able to perform very sophisticated movements compared to humans. Therefore, the RoboCup Humanoid league only differentiates between having physical contact (independent of the involved body parts) or not. In general, physical contact is allowed but needs to be minimized. Enduring contact must be avoided and leads to an intervention of the referee. The rules of other robot soccer leagues are similar, but might specify different periods and intensities of contact.

This indifference between the kinds of contacts becomes obvious when examining matches in the Humanoid Kid-Size league, especially the 2008 final between Nimbro and Team Osaka. Within this eventful 3 vs. 3 match, many physical interactions occurred. But in contrast to the variety of interactions in human soccer, which are described in the following section, only one reoccurring pattern can be observed: robots have contact, lose their balance, and fall over. The intensity of the impact with the floor is in any case disproportionately higher than any previous contact with any robot trunk or limb.

Because of this state of the art, dealing with different kinds of physical interactions (active or passive) to prevent damages, has not been addressed in the RoboCup community so far.

\subsection{Physical interaction in human soccer}

In this section, we classify physical interactions occurring in soccer (Fig. 4) and discuss their injury potential for the human and the robot. The discussion is based on a recent paper [43]. There we have analyzed scenes from the FIFA world championship 2006 in detail pretending that one of the players was a robot. We will now draw rather general conclusions for the robots design and behavior therefrom.

Tripping and getting tripped up. Tripping at high speed over the opponent's legs (Fig. 4a) seems to have a quite high injury potential and is a commonly observed action. It is not necessarily an intended foul, but can be a legal tackling which aims at the ball. Such interactions can cause fractures of extremities, ankle or knee injuries by direct contact [46] or indirectly from resulting tumble. Soft covering of the robotic leg can decrease this injury potential dramatically and also protects the robot's structure. Because tripping can be a sudden situation with little time to actively react, an overall compliant covering of the robot seems to be required. This is because the robot could fall in a more or less arbitrary direction with an undefined impacting zone. Passive compliance in the joints can decrease potential danger by intrinsically decoupling impacting masses. This requires avoiding outstretched configurations, since joint compliance has no effect there and the Cartesian reflected inertia is vastly increasing. On the other hand, for preventing damage to the robot it is important to strictly avoid joint limits.

Trunk and head impacts. Trunk and head impacts occur often, in particular while running (Fig. 4b) or during a header. These impacts require the limitation of the robot's weight because kinetic energy is, according to [47,48], a (limited) indicator of head injury and is at least somewhat related to chest injury. Therefore, the robot's weight has to be similar to the one of professional soccer players. This was also demanded by Burkhard et al.: "The robots should have heights and weights comparable to the human ones (at least for safety reasons) [...]" [49]. Current humanoid robots are less heavy but also smaller than the average soccer player [43]. The injury potential for a human being clamped on the ground by a robot that outweighs him is apparent. Such a situation poses significant danger to the limbs, chest, and other body parts.

Apart from limiting the robot weight, its body surface should definitely be padded to avoid human injuries from sharp edges, resulting in fractions, lacerations or cuts which already occur at blunt impacts [46]. Nevertheless, one should keep in mind that headers require a hard contact surface to accelerate the ball fast enough, therefore making a thinner coating for the head necessary. Possibly non-breakable materials such as rubber, polyurethane or silicone are the ones of choice.

Limb impacts. Dangerous impacts with the opponent's arm occur frequently and usually at the elbow (Fig. 4c). These impacts can actually be reduced to subhuman injury level by padding the robot's elbow (Section 3.4).

Impacts with the opponent's legs are usually with the boot which is the same for robots and humans. A particular problem is a lying goal keeper who could be fatally injured by kicking his head (Section 5.2). Such an accident could happen if the robot mistook the head for the ball. This makes a computer vision based ball detection software safety critical. Impacts with other parts except the head can again be eased by passive compliance in the joints, by decoupling the impact area from the rest of the robot, and by avoiding outstretched configurations.

Being hit by the ball. Being hit by a fast soccer ball can be a very painful and sometimes dangerous experience. In order to analyze such an impact, we carried out a one-dimensional simulation. The human head is modelled as a simple mass and the ball as a mass-spring system without damping, justified by high-speed camera recordings (see Fig. 5, top). Injury severity is expressed by the so-called Head Injury Criterion (HIC), following the extended Prasad/Mertz curves ${ }^{2}$ for the conversion to probability of injury. This criterion is the most important severity index for the head, e.g. used in automobile crash testing, biomechanics, and forensics

\footnotetext{
2 There exist various mappings to injury probability and interpretations of the HIC leading to different numerical values. However, we use one of them to show its extreme velocity dependency.
} 

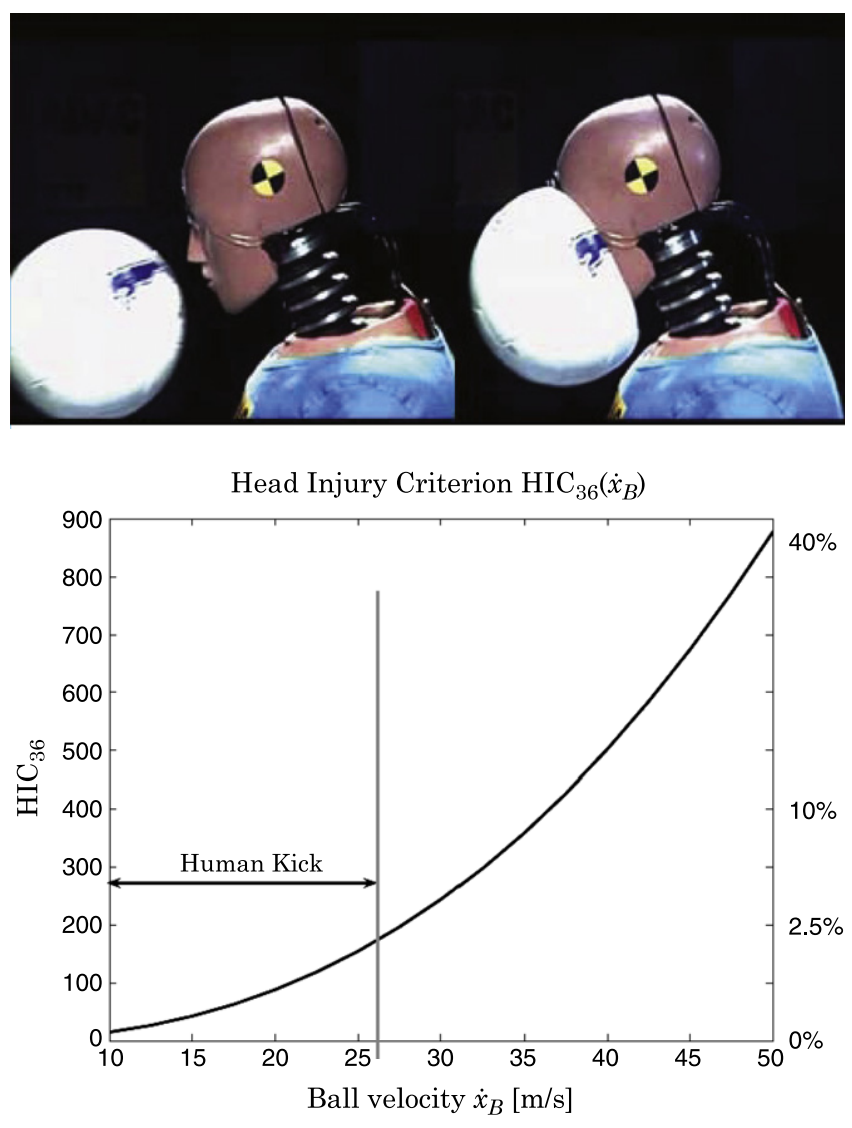

Fig. 5. Top: Hitting a Hybrid III dummy with a soccer ball. The impact is almost fully defined by the properties of the ball. The elasticity of the head can be neglected (courtesy of the German Automobile Club (ADAC)). Bottom: The HIC as a function of impact velocity and resulting probability $p($ AIS $\geq 3$ ) of serious (AIS $=3$ ) injury.

and was introduced to robotics in [37,19]. In Fig. 5 (bottom), the resulting Head Injury Criterion is plotted against impact velocity and the probability of serious ${ }^{3}$ injury for different impact velocities is indicated. It shows that a ball kicked by a human generally does not pose a serious threat, whereas increasing ball speed by only $50 \%$ would be already much more dangerous. These observations strictly forbid to compensate lack of robot intelligence by simple power, i.e. no "brute force" is possible in robot soccer.

In the following, we will outline how soft-tissue injuries and injuries caused by elbow checks can be reduced. Under certain circumstances it is even possible to limit them to lower levels than presumably caused by humans.

\subsection{How to avoid injuries from blunt impacts with soft tissue}

In order to analyze the benefit of intrinsic joint compliance, we will now evaluate the soft-tissue impact of a rigid robot joint with the lower abdominal area and then outline how decreasing the stiffness results in significantly improved safety characteristics. A main benefit of intrinsic joint compliance is that it gives a physical collision detection mechanism more time to detect and react to the collision since it decouples motor and link inertia. Before presenting the impact results, a short assessment of abdominal injury will be given to introduce a relevant injury severity index for the abdomen.

\footnotetext{
3 An internationally established classification of injury severity is defined by the Abbreviated Injury Scale (AIS) [50]. Serious injury is indicated by AIS $=3$.
}

The abdomen is located between the thorax and the pelvis. There exists a large literature on abdominal injury describing various different injury criteria with an overview given in [51]. For simplicity, we choose the side force criterion used in the EuroNCAP crash test. It states that the contact force must be

$F_{\text {ext }} \leq 2.5 \mathrm{kN}$.

This criterion will be used with a mass-spring system as a very simple model of the lower abdomen. The spring stiffness can be estimated from data published in [52] and is $K_{\mathrm{Abd}}=20 \mathrm{kN} / \mathrm{m}$. It will be assumed that the impact involves only the torso with a weight of $34 \mathrm{~kg}$ [42].

We simulate a kick with a hypothetical, faster version of the DLR-LWRIII at $7.5 \mathrm{~m} / \mathrm{s}$ which is clearly above any velocity common in human-robot interaction but reasonable for a soccer game. The reflected inertia of the motor and link are $13 \mathrm{~kg}$ and $4 \mathrm{~kg}$. In the following analysis, we will vary the joint stiffness from very rigid to fully compliant. ${ }^{4}$ An important feature of a robot interacting with its environment is a collision detection and reaction mechanism. We will show how such a mechanism together with intrinsic joint compliance significantly reduces the potential injury risk during a robot-human impact.

In Fig. 6, the contact force of a typical instep kick into the abdomen is shown with and without collision detection (left column), while on the right column the effect of joint damping is depicted. In current variable stiffness joints, physical joint damping is usually undesired [22], because it introduces hysteresis and possibly nonlinear behavior. However, human joints clearly are damped and therefore we show some properties related to damped joints as well.

For a very stiff robot, such as a typical industrial robot, the impact force results from an immediate impact of both, link and motor inertia acting basically as one interconnected mass. The limit force of the abdomen is clearly exceeded and therefore such an impact poses a severe threat to the human. In the case of a flexible joint robot as the DLR-LWRIII, the joint stiffness is already low enough to partially decouple link and motor inertia. The latter becomes significant approximately $50 \mathrm{~ms}$ after the link impact. This reduces the maximum force and gives a collision detection mechanism time to react. Due to the low link inertia, the first force peak is clearly below the tolerance force of the lower abdomen. For even lower joint stiffness (VIA "stiff" preset and VIA "soft" preset), both components are more and more decoupled and the delay of the second peak increases (caused by the much slower increasing joint force). This property would give an even less sensitive collision detection scheme time enough to react.

In order to show how effectively collision detection and reaction could reduce the impact forces caused by the influence of the motor, a collision detection and reaction is analyzed as well in Fig. 6 . The robot reacts to the detected impact by braking with maximum motor force as soon as a the collision is observed. For a very compliant robot, there is only the first impact peak left. However, already for a joint stiffness comparable to the one of the DLR-LWRIII, the height of the larger second peak can be diminished to a similar level as the first one.

Introducing joint damping $D_{J}$ has an interesting influence on the impact characteristics. For a flexible joint robot, motor and link inertia show less decoupling than that for the undamped case. However, the maximum value of the force is attenuated compared to the entirely stiff robot. For a VIA system, the damping leads to a larger joint force which decreases the effect of the motor inertia during the second peak. This way, the potential threat to the abdomen is fully eliminated even without any collision detection mechanism.

\footnotetext{
4 The problem of impacting in pretensioned state is not part of this analysis.
} 

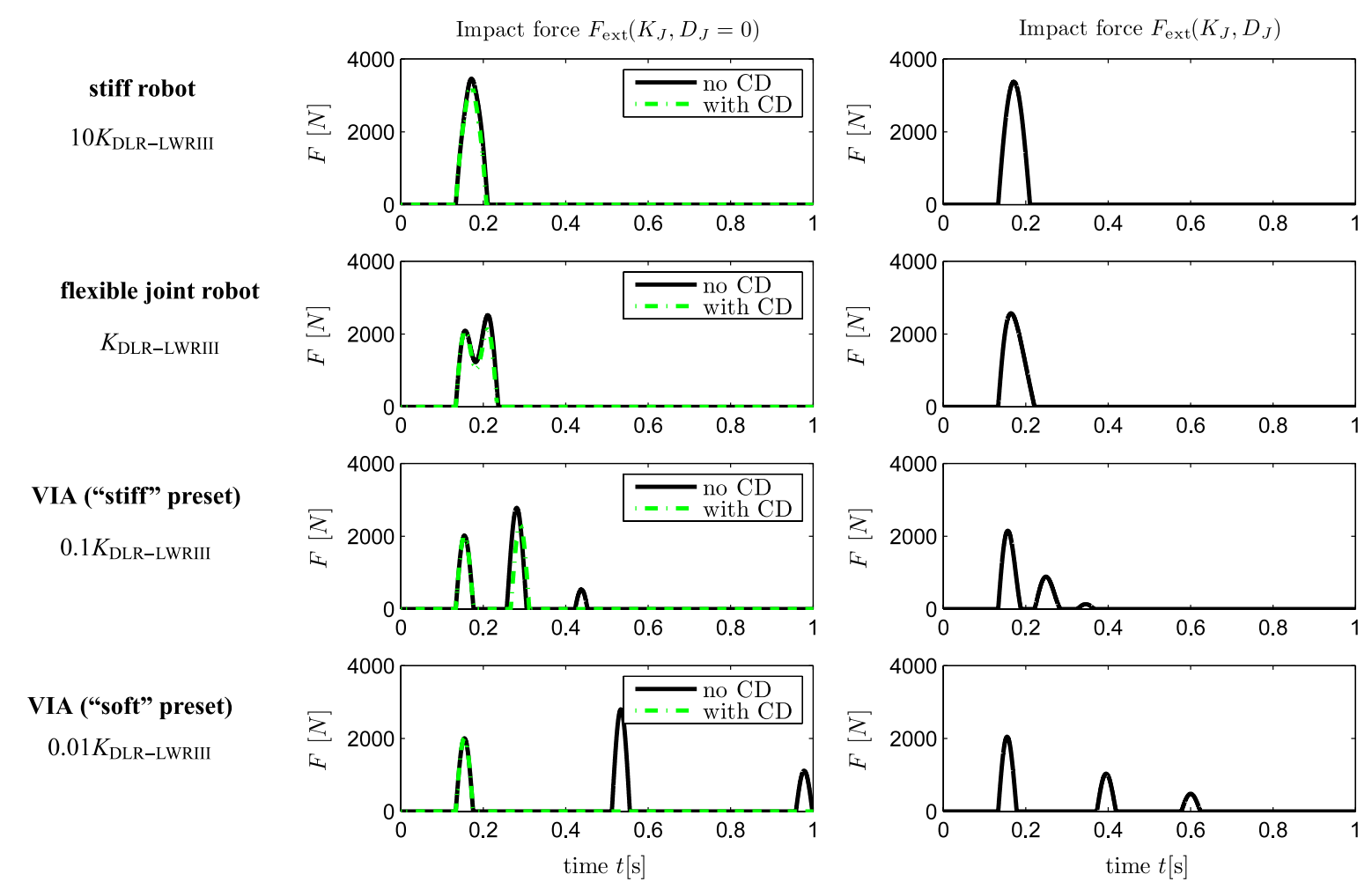

Fig. 6. Impacting the abdomen at $7.5 \mathrm{~m} / \mathrm{s}$ with a robot. The inertial parameters of the robot are the ones of the DLR-LWRIII and the joint stiffness is chosen to be $10,1,0.1,0.01$ times the one of the DLR-LWRIII. In other words, the compliance varies from very stiff to very compliant. The left plots show a robot without joint damping with and without collision detection (CD). If a collision is detected, the robot reacts by braking with full available motor force. The right plots show a critically damped link. For an intuitive understanding of this simulation please see the video provided at www.robotic.dlr.de/competitiveRobotics/.

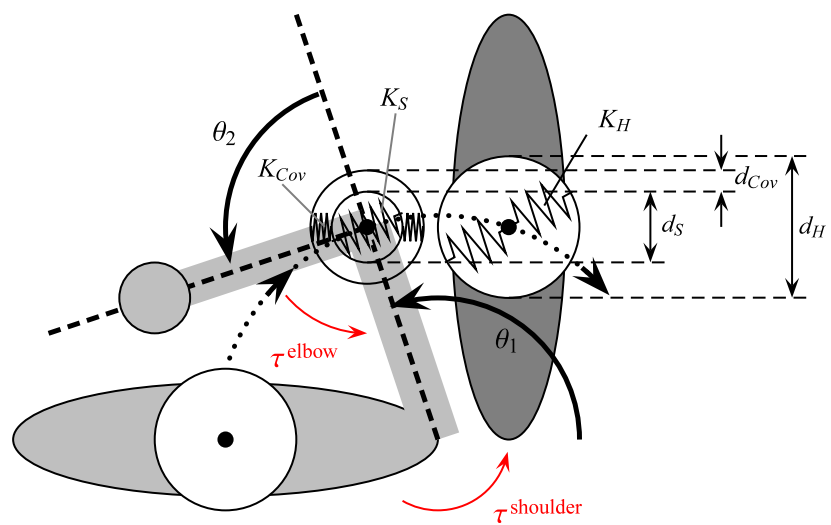

Fig. 7. Two-dimensional modelling of an elbow check. The left player hits the right player with his elbow on the head. The elbow is adjusted such that it produces the worst-case impact force for each setting. View from above.

\subsection{The elbow check: A frequent injury source in soccer}

According to [53], in elite football $41 \%$ of head injuries result from collisions with the elbow, arm, or hand of the opponent. In this section, simulation results will point out how dangerous elbow checks generally are. However, we will show that this threat can be reduced to lower levels than presumably caused by humans and even facial fractures can be prevented at all.

Fig. 7 shows the model. The human is represented as a mass-spring system, with a head mass of $4 \mathrm{~kg}$ [42], a contact stiffness of $K_{H}=10^{5} \mathrm{~N} / \mathrm{m}$ (maxilla, i.e. upper jaw [38]), and a fracture force of $660 \mathrm{~N}$ [54-56]. The arm/robot that is carrying out the elbow check is represented as a rigid body system with inertial parameters of the human arm [42]. The contact stiffness $K_{S}$ of the robot structure is modelled as the human elbow stiffness which is $K_{S}=7 \times 10^{5} \mathrm{~N} / \mathrm{m}$ during quasi-static bending [57].
In [58], elbow to head impacts were evaluated with human soccer volunteers and a HIII-Dummy. Impact velocities were $1.7-4.6 \mathrm{~m} / \mathrm{s}$. Hence we chose an impact velocity of $3 \mathrm{~m} / \mathrm{s}$ and assumed here that the involved players have no relative velocity during the incident. We also chose the worst elbow angle of $\theta_{2}=$ $\frac{\pi}{2}$ (Fig. 7). The maximum human shoulder and elbow torques according to [59] are

$\left(\left|\tau_{\max }^{\text {shoulder }}\right|,\left|\tau_{\max }^{\text {elbow }}\right|\right)=(80,60) \mathrm{Nm}$.

These are calculated from analyzing baseball pitches during a throw. In order to show the improvement adequate covering could have, the influence of covering thickness and material type on the contact force are analyzed in Fig. 8. The elasticity modulus $E_{\mathrm{cov}}$ of the covering was chosen to range up to rather hard rubber and its thickness increases up to $d_{\text {cov }}=0.15 \mathrm{~m}$.

Without any countermeasure the contact force easily exceeds the fracture tolerance of the human maxilla (Fig. 8). On the other hand, with a collision detection and reaction scheme similar to the ones introduced in $[60,61]$, it is possible to reduce impact forces significantly, even without any covering $\left(d_{\mathrm{Cov}}=0 \mathrm{~m}\right)$ by $\approx 150 \mathrm{~N}$. Compliant covering is the second very effective approach to reduce dynamic impact forces. Of particular interest is that for each covering thickness an optimal value for the elasticity modulus exists (Fig. 8, right).

In the simulation, it seems that a good collision detection and reaction scheme is almost as effective in reducing impact forces as providing thick covering. In reality, this is of course limited by the motor dynamics and the resulting motor torques (joint torques in the flexible case). Furthermore, detection delays and system latencies need to be considered which additionally lower the absolute effectiveness. 

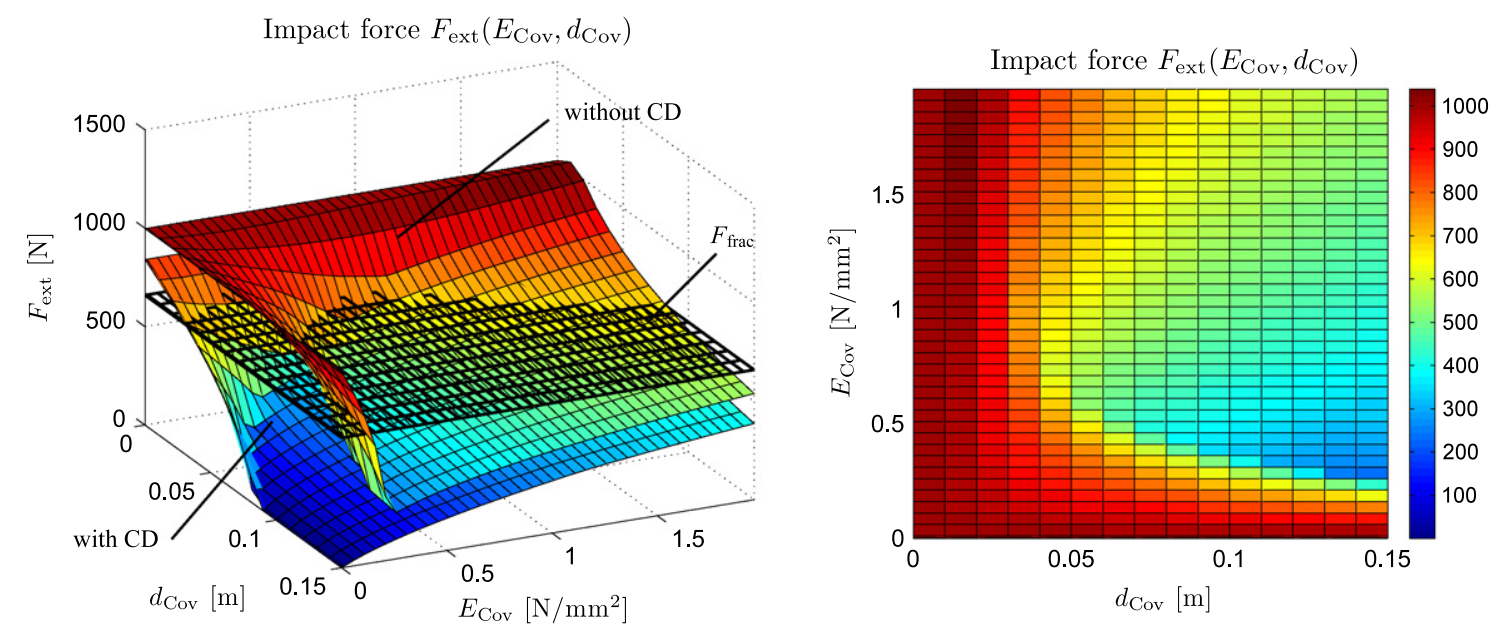

Fig. 8. Left: Force as a function of covering elasticity modulus and thickness for an elbow check with the maxilla (upper jaw) at $3 \mathrm{~m} / \mathrm{s}$. CD indicates whether a collision detection and reaction scheme is activated or not. The reaction consists basically of rapidly "fleeing" from external forces. Right: It becomes clear that (without CD) there exists for each specified covering thickness $d_{\mathrm{Cov}} \geq 4 \mathrm{~cm}$ an optimal material which is able to provide impact forces below the fracture tolerance.

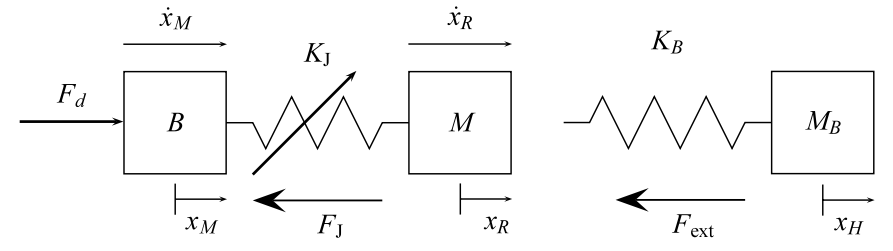

Fig. 9. One-dimensional model of kicking a soccer ball with a variable stiffness robot. The robot is modelled as a mass-spring-mass system, representing the motor mass, joint stiffness, and link mass with $B=13 \mathrm{~kg}, M=4 \mathrm{~kg}$, and $K_{\mathrm{J}} \in\{130,1300,13000\} \mathrm{N} / \mathrm{m}$. The ball is modelled as a mass-spring element with $M_{B}=0.45 \mathrm{~kg}$, and $K_{\text {Ball }}=43.7 \mathrm{kN} / \mathrm{m} . B, M$ were selected to be the reflected inertias in the case of a typical stretched out collision configuration with the DLR-LWRIII.

\section{PART II: Protecting the robot by joint compliance}

In this part, a trend in physical Human-Robot Interaction is discussed that led to the development of novel joint designs incorporating mechanical joint compliance [62] or even variable stiffness actuation (VSA). As mentioned in Section 2, various control schemes to realize compliance by means of active control are described in the literature. However, motion in sport happens at extreme joint velocities, e.g. $1375 \mathrm{deg} / \mathrm{s}$ for instep-kicking [44] or even 6900-9800 deg/s during a baseball pitch [59]. At such velocities, it seems very unrealistic to achieve compliance by control, since results in [63] indicate a limit already at much lower velocities for a state-of-the-art robot. A particular reason for that is actuator saturation. In this section, we focus on the situation of an external impact. For a stiff joint, the motor has to immediately follow that impact, leading to an extreme torque that can damage the gears. We observed this effect during impact experiments with the DLR-LWRIII reported in [5]. Since the torque is much higher than what the motor can generate, this problem cannot be solved by control but only by mechanical compliance in the joint.

\subsection{The relationship between joint stiffness and kicking force}

In order to visualize the effect of joint elasticity on the joint force, we simulated a one-dimensional example (see Fig. 9). It outlines the dramatic decrease of joint force during an impact with a soccer ball at $\dot{x}_{R} \in\{2,4,10\} \mathrm{m} / \mathrm{s}$ for a variable stiffness joint. In Fig. 11, the impact forces are given, showing that even with reduced joint stiffness they basically stay the same at different kicking velocities. This again is due to the decoupling of link and motor inertia happening already at a high stiffness.
Concerning the load on the joint, one can see that although the contact force $F_{\text {ext }}$ stays the same, the joint force $F_{\mathrm{J}}$ decreases dramatically for a joint stiffness reduced by one or two orders of magnitude compared to the DLR-LWRIII. A full-robot simulation of this phenomenon is documented in [43]. So one can say that more elasticity helps protecting robot and human but for the human a benefit can be seen only up to the point where motor and link become practically decoupled.

Now an experimental evaluation of a new variable stiffness joint prototype [22] is going to be discussed with the aim of quantifying the achievable gain in joint protection.

\subsection{Kicking a soccer ball against the DLR VS-Joint: Experiments}

There are generally two main approaches to realize variable joint compliance. The first one is the biologically motivated antagonistic concept using its two actuators for both, position and stiffness adjustment. The second one is to assign one actuator mainly for positioning and the other one for changing the joint stiffness. However, most conclusions made in this paper can be generalized to both types. The prototype used in this paper is of the second type and its basic concept is visualized in Fig. 10. The positioning motor is connected to the link via a harmonic drive gear. Mechanical compliance is introduced by a mechanism which forms a flexible rotational support between the harmonic drive and the joint base. In the case of a compliant deflection of the joint caused by the external torque, the entire harmonic drive gear rotates relative to the base but at the same time the positioning motor does not change its position.

The effect of joint stiffness on the resulting joint torque of the DLR VS-Joint prototype is investigated during impact loading with a soccer ball. When kicking or throwing a ball against the link, it is hard to reproduce impact position and velocity. Therefore, instead of kicking the ball, the entire setup is moved along a trajectory and hits the soccer ball at a constant velocity. This was done by mounting the setup upside down on the Tool Center Point (TCP) of a KUKA Robocoaster (see Fig. 12). This robot weighs $2500 \mathrm{~kg}$ and can therefore be treated as a velocity source during the following analysis. In this setup, maximum horizontal velocity is achieved by moving the Robocoaster in an "outstretched" configuration at maximum velocity in its first joint. A wooden shoe-tree in a standard football shoe is attached to the tip of the joint lever. The joint torque $\tau_{J}$ is measured $\left(\tau_{\mathrm{msr}}\right)$ with a strain gauge torque sensor at the base of the link lever. Furthermore, the joint motor position $\theta$ and the link lever position $q$ are measured by rotational encoders. 


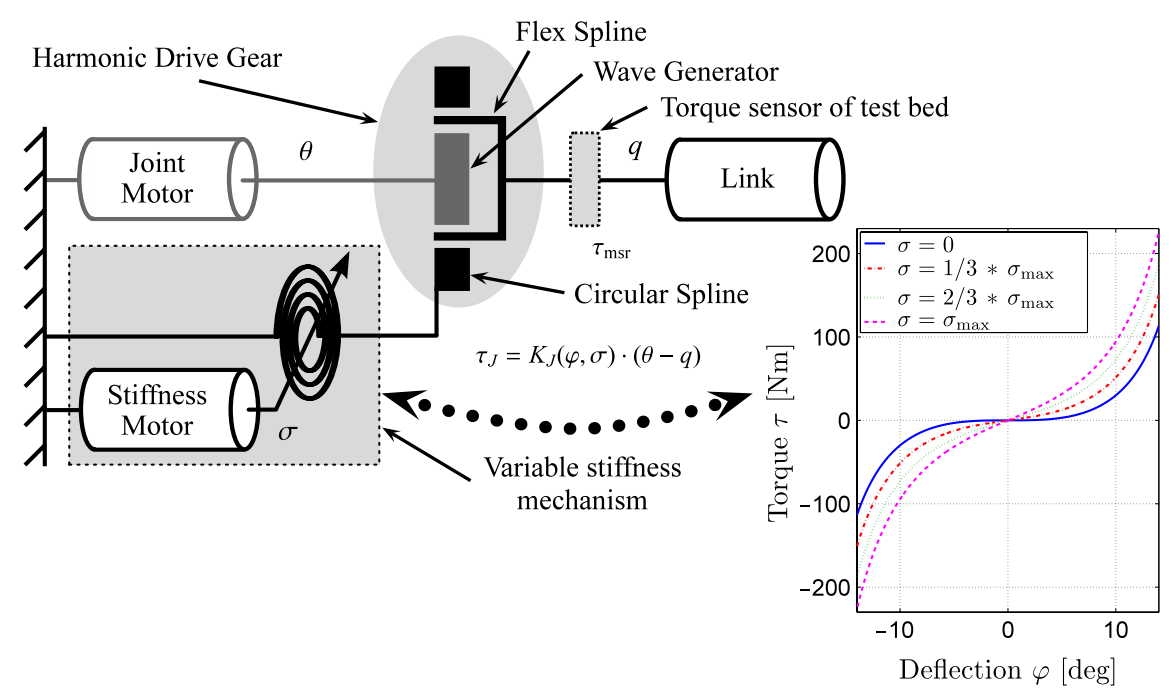

Fig. 10. Principle of variable stiffness joint mechanics. The circular spline of the harmonic drive gear is supported by the VS-Joint mechanism.
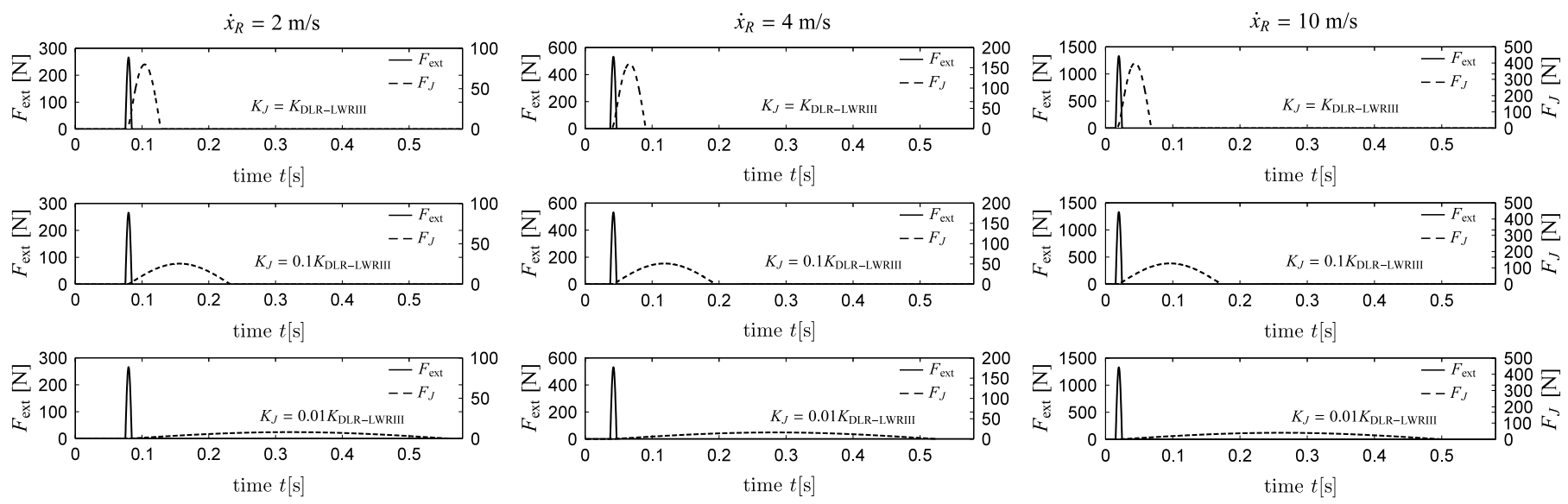

Fig. 11. Simulation describing the effect of stiffness reduction on impact force and spring force for a kicking velocity of $2 \mathrm{~m} / \mathrm{s}, 4 \mathrm{~m} / \mathrm{s}, 10 \mathrm{~m} / \mathrm{s}$. The solid line indicates the contact force and the dashed line indicates the spring force. The spring force decreases in magnitude and increases in duration when reducing the spring stiffness, whereas the contact force basically stays the same for each particular impact velocity. These are results from the simulation explained in Section 4.1 .

The difference between both is the passive joint deflection $\varphi=\theta-q$. The impact configuration was an instep kick (see Section 5.3).

The impact tests were carried out at four different impact velocities and with three parameterizations of the torquedeflection function ${ }^{5}$ (see Fig. 13). Two stiffness setups are realized via the passively compliant VS-Joint. We chose the most compliant as well as the stiffest configuration $\left(\sigma=0\right.$ and $\left.\sigma=\sigma_{\max }\right)$. Depending on the joint deflection, the corresponding stiffness is ranging from $0 \mathrm{Nm} / \mathrm{deg}$ to $37 \mathrm{Nm} / \mathrm{deg}$ in the compliant and from $5.5 \mathrm{Nm} / \mathrm{deg}$ to $55 \mathrm{Nm} / \mathrm{deg}$ in the stiffest configuration. In the third setup, a mechanical shortcut is inserted into the testbed instead of the VS-Joint mechanism, leading to a rather stiff intrinsic behavior of $\approx 520 \mathrm{Nm} / \mathrm{deg}$. The numerical value is in the range of the DLR-LWRIII elasticity in the first joint which is $\approx 350 \mathrm{Nm} / \mathrm{deg}$.

Both, increasing impact speed and joint stiffness result in higher peak joint torques as visualized in Fig. 13 (top). The maximum peak torque limit of the joint gear is almost reached with the stiff joint at an impact velocity of $\approx 3.7 \mathrm{~m} / \mathrm{s}$, whereas the compliant VS-Joint is still far in the safe torque region.

\footnotetext{
5 The joint stiffness $K_{J}\left(\varphi, \sigma^{*}\right)=\frac{\partial \tau\left(\varphi, \sigma^{*}\right)}{\partial \varphi}$ for some stiffness preset $\sigma^{*}=$ const. is a highly nonlinear function as can be observed in Fig. 13.
}

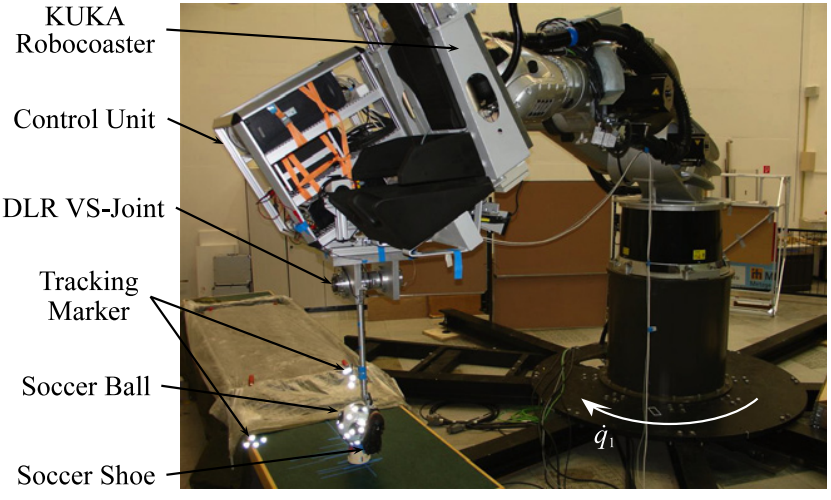

Fig. 12. Test setup for hitting the VS-Joint with a soccer ball. The testbed for the VS-Joint is mounted upside down on a KUKA KR500/Robocoaster. The entire joint testbed is moved horizontally with a constant Cartesian velocity of up to $3.7 \mathrm{~m} / \mathrm{s}$ by the KR500. The link hits the resting ball in non-pretensioned state with an attached foot that is equipped with a standard soccer shoe (see Fig. 16). This allows us to investigate the effect of the resting joint being hit by a ball in a controlled and reproducible environment.

During the impact, a certain amount of kinetic energy is transferred to the joint. Apart from parasitic effects such as friction 

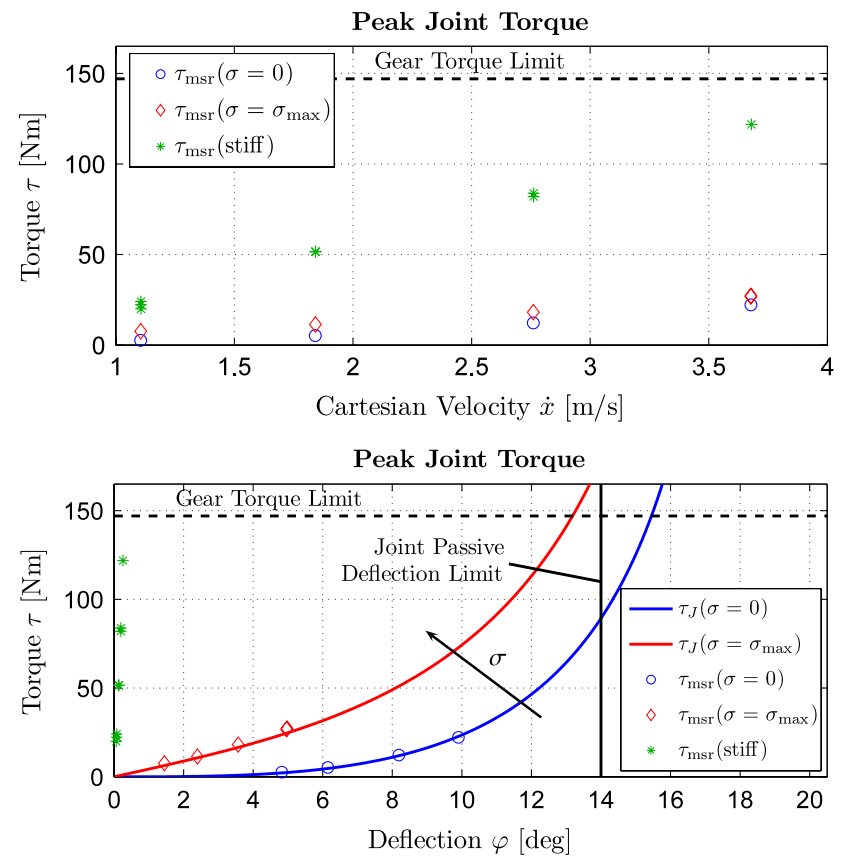

Fig. 13. Top: Peak joint torque during impacts with a soccer ball and the VS-Joint. The impact velocity ranges up to the maximum velocity of the KR500/Robocoaster. Three different stiffness setups are examined: VS-Joint at low stiffness preset, VS-Joint at high stiffness preset, and an extremely stiff joint without deliberate elasticity. Bottom: Peak joint torque during impacts of a soccer ball on the soccer foot mounted on the joint. Higher impact velocities result in larger peak torque and passive joint deflection. At the same speed a soft joint stiffness preset $(\sigma=0)$ causes significantly lower joint torque but higher joint deflection. Therefore, a very soft joint faces a higher risk of running into the deflection limits. For a very stiff joint, the gear torque limit poses an upper bound for the maximum impact velocity. Maximal two trials were carried out for each velocity and stiffness configuration.

and damping, the complete transferred energy is stored at a certain moment as potential energy in the joint spring. Increasing impact velocity naturally enlarges the amount of transferred energy. This, in turn, results in increased joint deflection during the impact (see Fig. 13, bottom). If the compliant joint has a maximal passive deflection angle, this poses a second safety limit to the joint. Therefore, one needs a trade-off: On the one hand, lower stiffness results in lower peak torques but higher joint deflections and one may run into joint limits. On the other hand, higher stiffness causes higher peak torques and may damage the gears or the structure of the joint itself. The stiffness has to be chosen such that both limits are avoided, if possible.

The preceding evaluation outlined how joint elasticity can effectively reduce high impact joint torques and the related risk of joint damage. In the following, we will investigate the ability of a VSA to use its inherent physical elasticity as an energy storage and release mechanism. This feature is especially powerful for achieving very high link speeds, which in turn are necessary to be able to kick a soccer ball strong enough.

\section{PART III: Increasing robot performance by elastic joints}

For future soccer robots, kicking a ball at human speed level is a major requirement in order to be a serious opponent to their human counterparts (Fig. 14, left).

This part discusses, how joint elasticity can be used to close the large gap in joint velocity between current robots and human soccer players [44]. A general argument in favor of intrinsic joint compliance is its ability to store and release energy

1. for decreasing the energy consumption of the system or

2. to increase peak power output.
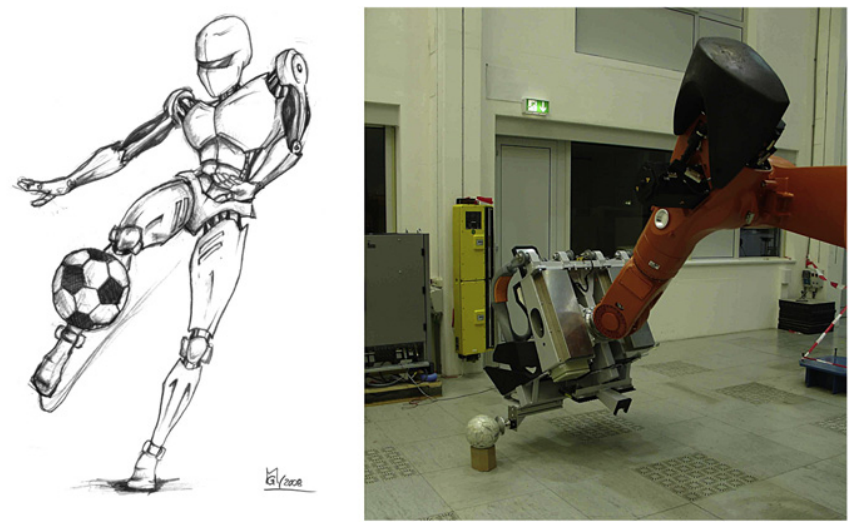

Fig. 14. Left: Kicking a soccer ball at high impact speed. Right: A football kick with a KUKA KR500 weighing $2500 \mathrm{~kg}$ at maximum velocity. The reflected inertia during such an impact is $1870 \mathrm{~kg}$.

The former has received larger attention especially for biped walking $[31,32,17]$. Our focus lies on the latter as it allows us to considerably increase the link speed $[64-66,43,22]$ above motor level.

\subsection{Kicking in RoboCup}

For comparing the results presented in this paper with the performance of current soccer robots, a short overview of the state of the art regarding ball manipulation abilities in RoboCup is given in this section.

The currently largest and most powerful - by means of joint torque - humanoid soccer robots play in the Humanoid Teen Size League. In this league, an orange beach handball (size 2; $18 \mathrm{~cm}$ diameter, weighing $294 \mathrm{~g}$ ) is used [45]. The robots have to manipulate the ball using their legs. In most cases, a humanoid leg is constructed as a sequence of six joints which allow - in addition to kicking - omnidirectional walking patterns. The 2007 world champion, team NimbRo from Freiburg [67], powered these joints with Dynamixel RX-64 servo motors (as several other teams do), which have a holding torque of $6.4 \mathrm{Nm}$ and a maximum velocity of about $360 \mathrm{deg} / \mathrm{s}$ (specification from manufacturer) without load. By coupling pairs of these motors in several joints of their robot Robotina, the torque is doubled. The knees of this robot are additionally supported by torsional springs. Robotina is able to kick the standard ball at a velocity of about $2 \mathrm{~m} / \mathrm{s}$ but cannot lift it from the ground significantly.

\subsection{The joint velocity required}

In the following, we will calculate the joint velocity necessary for kicking a ball with the DLR-LWRIII at a speed comparable to a human instep kick. According to [2], the velocity of the ball can be expressed accurately enough by

$\dot{x}_{B}=\dot{x}_{F} \frac{m_{F}(1+e)}{m_{F}+m_{B}}$,

where $m_{F}=m_{B}=0.45 \mathrm{~kg}$ and $e \approx 0.5$. All symbols are defined in Table 2. Since the DLR-LWRIII has in outstretched position a reflected inertia of $\approx 4 \mathrm{~kg}$ along the impact direction, the velocity of the robot's end needs to be $\approx 0.75 \dot{x}_{B}$, leading with $16 \mathrm{~m} / \mathrm{s} \leq$ $\dot{x}_{B} \leq 27 \mathrm{~m} / \mathrm{s}$ for real kicks to

$12 \mathrm{~m} / \mathrm{s} \leq \dot{x}_{F} \leq 20.25 \mathrm{~m} / \mathrm{s}$. 


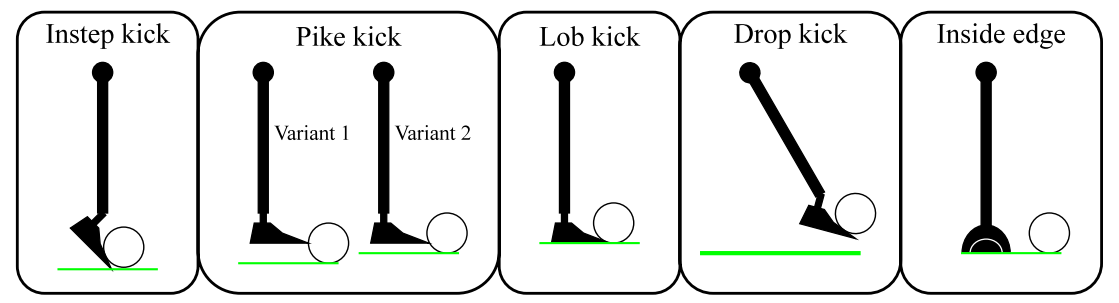

Fig. 15. Kicking techniques investigated in the framework of this paper. Only the drop kick allows a foot position below the ball.

This corresponds to a joint velocity of $414 \mathrm{deg} / \mathrm{s}$ to $700 \mathrm{deg} / \mathrm{s}$, much higher than the maximal joint velocity of the DLR-LWRIII $(130 \mathrm{deg} / \mathrm{s})$. Due to the smaller reflected inertia of a human foot, humans kick at an even higher joint velocity of up to $1375 \mathrm{deg} / \mathrm{s}$ for knee extension and with joint torques up to $280 \mathrm{Nm}$ [44]. Kicking a soccer ball at the maximum nominal joint velocity of the DLR-LWRIII leads to a ball velocity of $\approx 4.5 \mathrm{~m} / \mathrm{s}$, i.e. six times slower than required. Even with such a low velocity, the joint torques already become critical (80\% of maximum nominal torque) [43]. This is confirmed by observations we made during robot-dummy impacts presented in [5], where the exceedance of maximum nominal joint torques was observed already at impact velocities of $\approx 1 \mathrm{~m} / \mathrm{s}$.

\subsubsection{Kicking with a heavy-duty industrial robot}

In order to show by a very intuitive experiment the performance limits of classical actuation, a soccer ball was kicked with a KUKA KR500, one of the world's largest robots (500 kg payload) weighing almost $2500 \mathrm{~kg}$. Maximum joint velocity results in an impact at $3.7 \mathrm{~m} / \mathrm{s}$ (Fig. 14, right). Still the ball hits the ground after a flight of only $\approx 2 \mathrm{~m}$. This example gives a good feeling about the large gap in joint velocity between current robots and the RoboCup 2050 challenge and especially supports the claim that increasing robot mass does not significantly enhance kicking performance.

\subsection{Kicking a ball with an elastic joint}

The recent example of Asimo, currently one of the fastest biped humanoid robots, or the successful robots of Humanoid Team NimbRo kicking a soccer ball reveal a large gap in the kicking performance between current humanoid robots and humans. In this part of the paper, we will show how much higher kicking performance is achievable already with a single elastic joint. Of course, this experiment is not meant as an assessment but to show the potential of elastic joints. Our joint, the new DLR VSJoint is equipped with an adjustable passive elastic element which serves as an energy storage and release mechanism (see Fig. 10). It allows us to significantly increase the link speed as pointed out and analyzed to some extent in $[64,66,43,65]$. In order to show that the proposed increase in kicking performance is not only achievable for a particular type of kicking, we conducted experiments with five basic kicking techniques shown in Fig. 15.

\subsubsection{Kicking test setup}

In this paper we will evaluate the most common kicking techniques used in soccer: instep, pike, lob, and drop kick as well as inside edge pass. These techniques require appropriate foot angle setups (see Fig. 16). For this reason, the foot angle can be changed in two axes. The first axis is concentric to the joint lever. Its angle $\phi_{1}$ is set to 0 deg for all techniques except for the inside edge pass where it is set to $-90 \mathrm{deg}$. The second axis is rotated by 90 deg relative to the first axis and is parallel to the joint axis in the case of $\phi_{1}=0$. The angle $\phi_{2}$ of the second axis is changed according to the kick technique. The inertia of the lever and foot

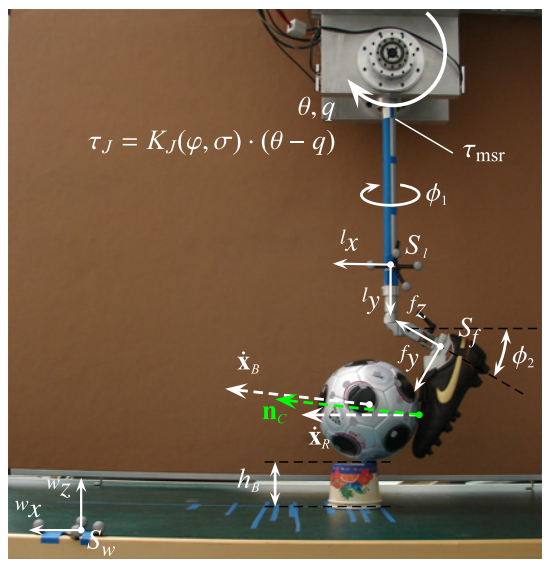

Fig. 16. Test setup for kicking a ball depicted for an instep kick. The testbed for the DLR VS-Joint is mounted upside down. The angle $\phi_{2}$ between the foot and the limb (joint lever) is altered by a hinge. The height of the ball $h_{B}$ is adjusted by the number of piled cups underneath and adjusted according to the investigated kicking technique (see Fig. 15). The normal on the contact point between foot and ball is denoted as $\mathbf{n}_{C}$.

is $J \approx 0.57 \mathrm{~kg} \mathrm{~m}^{2}$ slightly depending on the foot orientation. The height $h_{B}$ of the ball can be changed to adjust the position of the contact point between ball and foot. A tracking system was used to track the position of the link $S_{l}$ and of the ball relative to a world coordinate system $S_{w}$. This is done by two 6-DOF markers mounted to the link and to the table respectively. The coordinate system $S_{f}$ was identified with the tracking system for each foot position relative to $S_{l}$. Furthermore, the surface of the shoe was sampled by grid points relative to $S_{f}$. This allows us to calculate the contact normal $\mathbf{n}_{C}$ between the foot and the ball out of the tracking data. The trajectory of the ball is also measured by the tracking system.

\subsubsection{Kicking trajectory}

The link velocity of a stiff joint is limited by the velocity of the driving motor. In a flexible joint, the potential energy stored in the system can be used to accelerate the link relative to the driving motor. Additionally, potential energy can be inserted by the stiffness adjuster of the variable stiffness joint.

In the experiments presented in this paper, a simple strike out trajectory is used (Fig. 17). A motor position ramp accelerates the link backwards to increase its kinetic energy. Then the motor reverts which in turn leads to a transformation of the kinetic link energy into potential energy stored in the VS-Joint spring. The stiffness adjuster starts moving with maximum velocity to the stiffest configuration, additionally increasing the potential energy of the system. The next step is to accelerate the motor up to its maximum velocity, adding kinetic energy to the VS-Joint. As soon as the link starts to catch up with the motor, its velocity increases up to the motor maximum velocity plus a term depending on the amount of the stored potential energy

$\dot{q}_{\max }=\dot{\theta}_{\max }+\dot{\varphi}_{\max }=\dot{\theta}_{\max }+\sqrt{2 J^{-1} E_{\max }(\varphi, \sigma)}$, 
Table 1

Results for the different kicks investigated given for the VS-Joint and for the entirely stiff joint.

\begin{tabular}{|c|c|c|c|c|c|c|c|c|c|c|}
\hline Type & Ball & Variant & \# trials & $\phi_{2}$ (deg) & $\dot{q}(\mathrm{deg} / \mathrm{s})$ & $\dot{x}_{R}(\mathrm{~m} / \mathrm{s})$ & $F_{\text {ext }}(\mathrm{N})$ & $x_{\text {kick }}(\mathrm{m})$ & $\dot{x}_{B}(\mathrm{~m} / \mathrm{s})$ & $h_{\text {kick }}(\mathrm{m})$ \\
\hline Instep kick & Football & Stiff & 1 & 30 & 228 & 3.05 & 144 & - & - & - \\
\hline Instep kick & Football & VSA & 3 & 30 & 498 & 6.65 & $343-359$ & - & $6.6-7.5$ & - \\
\hline Instep kick & Football & VSA & 4 & 45 & 490 & 6.56 & $387-0473$ & - & $6.0-7.0$ & - \\
\hline Instep kick & Football & VSA & 3 & 60 & 490 & 6.50 & 503-591 & $3.40-3.65$ & $5.7-6.0$ & - \\
\hline Pike kick & Football & Stiff $90 \mathrm{deg}$ & 1 & 90 & 231 & 3.09 & 141 & 0.60 & 3.0 & - \\
\hline Pike kick & Football & VSA $90 \mathrm{deg}$ & 3 & 90 & 489 & 6.20 & $447-503$ & $2.90-3.50$ & $8.0-10.0$ & - \\
\hline Pike kick & Football & Stiff $45 \mathrm{deg}$ & 1 & 90 & 226 & 3.02 & 111 & 1.43 & 5.0 & - \\
\hline Pike kick & Football & VSA $45 \mathrm{deg}$ & 3 & 90 & 489 & 6.20 & 548-640 & $3.20-3.40$ & $5.5-7.7$ & - \\
\hline Lob kick & Football & Stiff & 1 & 90 & 228 & 3.04 & 96 & - & 1.9 & 0.65 \\
\hline Lob kick & Football & VSA & 3 & 90 & 488 & 6.00 & $374-390$ & - & 3.9 & 0.84 \\
\hline Drop kick & Football & Stiff & 1 & 30 & 229 & 3.06 & 172 & 1.60 & - & - \\
\hline Drop kick & Football & VSA & 3 & 30 & 475 & 6.35 & $354-483$ & $3.80-4.05$ & - & - \\
\hline Drop kick & Handball & VSA & 3 & 30 & 477 & 6.37 & $389-419$ & $3.40-3.70$ & - & - \\
\hline Drop kick & RoboCup & VSA & 4 & 30 & 476 & 6.36 & 163-203 & $5.90-6.30$ & - & - \\
\hline
\end{tabular}

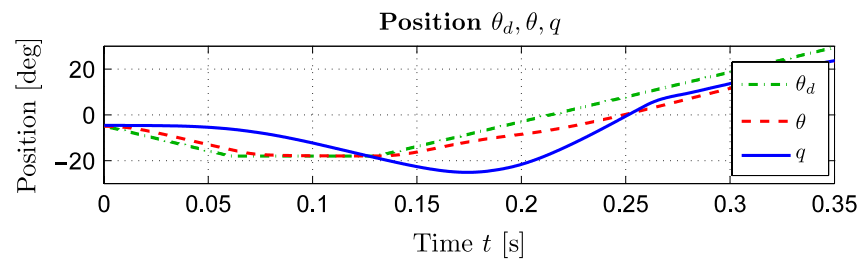

filtered to obtain the link acceleration and second, the radial force component cannot be calculated from torque signal.

For $\phi_{2}=30 \mathrm{deg}$ and $\phi_{2}=45 \mathrm{deg}$, it is not meaningful to measure $x_{\text {kick }}$ since the ball practically does not lift.

Kicking with the pike is mainly varied by the position at which the ball is hit. We only evaluated vertical variation, because horizontal variation causes spin and is left for future work. We investigated two impact positions which were chosen to be perpendicular to the ball surface (90 deg contact) and hitting the ball at an angle of $45 \mathrm{deg}$ ( $45 \mathrm{deg}$ contact). (see Fig. 15). The impact forces were generally higher compared to the instep kick and the kicking ranges are very large as well. This seems mainly to be caused by the rigid contact at the pike.

The lob is basically a pike kick hitting the ball as low as possible, generating a very smooth parabolic trajectory, lower ball velocities and contact forces. The main idea behind a lob is to kick the ball beyond the opponent (often the goalkeeper in a direct one to one situation). So one has to lift the ball rapidly very high. We were able to kick the ball such that it lifted $0.82 \mathrm{~m}$ at a horizontal travelling distance of $0.6 \mathrm{~m}$.

In order to compare the drop kick, we measured the kicking range with three different balls. Apart from the football, an indoor handball and a plastic RoboCup ball, used in the Standard Platform league, were evaluated. Each ball was hit such that it was contacted at a 45 deg angle. The ball velocities were lower than for the other kicks but at the same time we were able to shoot up to a distance of $4 \mathrm{~m}$ with a football and more than $6 \mathrm{~m}$ with the Robocup ball. The handball was not a beach handball as used in the Humanoid soccer league but an indoor version which is heavier $(0.45 \mathrm{~kg})$. It has basically the same weight as a soccer ball but apparently quite different contact characteristics which is presumably due to the different requirements from the sport itself (kicking vs. dribbling and throwing).

For the inside edge pass, the entire foot was rotated to $\phi_{1}=$ $-90 \mathrm{deg}$ and $\phi_{2}$ was set to $90 \mathrm{deg}$. Thus, we were able to kick the ball with the inside edge of the shoe. With this type of kick it is possible to kick the soccer ball the fastest so that it reached maximum velocities of $7.8-9.8 \mathrm{~m} / \mathrm{s}$.

While evaluating such a kick in terms of the physical parameters, as done so far, is straightforward, evaluating the effectiveness of such a kick seems to be a very difficult thing to do since it is absolutely depending on the game situation whether it was a success or fail.

After this evaluation of the kicking performance with different techniques we would like to point out a quite remarkable observation we made when comparing the drop kick of a stiff with a VS-Joint by means of speed, kicking range, and impact joint torque. Although the impact speed with a VS-Joint more than doubles and the kicking range can be more than three times 


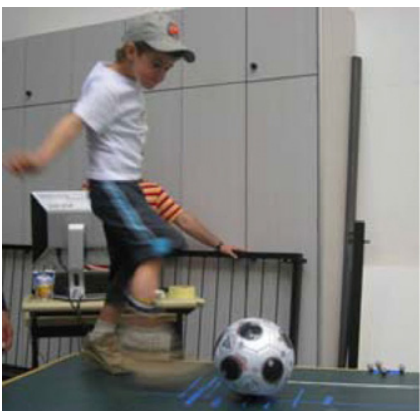

Fig. 18. Comparing the kicking abilities of a 5 year old boy with the DLR VS-Joint prototype. Position and velocity of foot and ball were tracked.

higher compared to a stiff joint, the impact joint torque during the observed kicks is only $10 \mathrm{Nm}$ for the VS-Joint in contrast to $85 \mathrm{Nm}$ for the stiff joint. This clearly shows that performance can be increased along with effective joint protection.

\subsubsection{Comparison with a human child kick}

Of course, it is not possible to shoot anywhere close to professional level or at least to an adult human kick with a singlejoint-setup. However, in order to compare performance as a showcase to a real human, we let a 5 year old boy kick with the soccer ball lying on the ground and on the same height as used for the instep kick (Fig. 18). The leg length of the child is shorter $(0.54 \mathrm{~m})$ compared to our prototype link length but he was allowed to kick as hard as possible without any restrictions on the used degrees of freedom.

The boy achieved ball velocities of $5-6 \mathrm{~m} / \mathrm{s}$, i.e. comparable to the ones we obtained with our setup. The kicking length range was $1.5-4.2$ m depending very much on the "quality" of the kick. The foot velocity was relatively constant $10-13 \mathrm{~m} / \mathrm{s}$ at the time instant of the kick, leading to the conclusion that the reflected inertia is significantly lower than for the setup.

To sum up, it can be stated that in all evaluated cases we were able to obtain very good kicking performance and the benefit of the intrinsic joint elasticity was clearly verified. It seems very promising to further evaluate the n-DOF case in the future.

\subsection{Optimal control for kicking with an elastic joint}

In this last section, we analyze theoretically, how much velocity can be gained from using (constant) joint elasticity and what is the price. We therefore consider a standard elastic joint model [68] with the motor acting as a pure velocity source. We do not consider geometrical constraints or nonlinear elasticity, because this would be rather complicated. The model is

$\dot{\theta}(t)=u(t), \quad|u(t)| \leq u_{\max }$

$\ddot{q}(t)=\frac{K_{J}}{J}(\theta-q)$

$q(0)=\dot{q}(0)=\theta(0)=\dot{\theta}(0)=0$

where $q$ is the joint position, $\theta$ the motor position, $K_{J}$ the joint stiffness, $J$ the link inertia, and $u$ the control command. Without damping, a mass-spring system can be excited to arbitrarily large oscillations. However, these need time to build up. So we ask what is the largest joint velocity that can be achieved within a time $T$ leading to an optimal control problem. To address this problem, we consider the closed solution of (6)-(8).

$\theta(T)=\int_{0}^{T} u(t) \mathrm{d} t$

$q(T)=\int_{0}^{T} u(t)(1-\cos (\omega(T-t))) \mathrm{d} t$

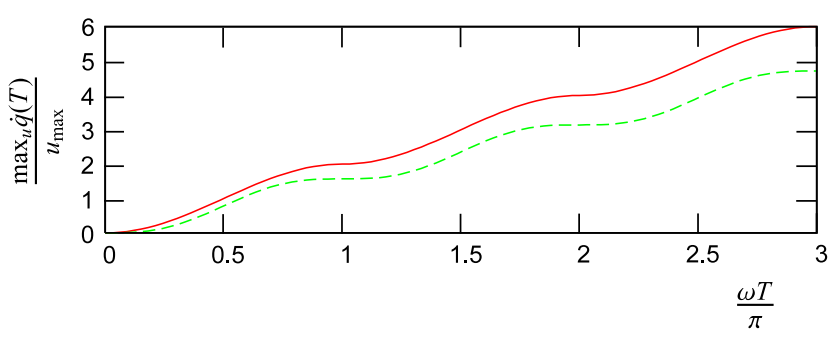

Fig. 19. The speedup achievable in time $T$. The $X$-axis indicates the time $\frac{\omega T}{\pi}$ in halfcycles of the spring-mass eigenfrequency. The Y-axis indicates the achievable joint velocity $\frac{\max _{u} \dot{q}(T)}{u_{m a x}}$ relative to the motor velocity. The top plot shows optimal bangbang control, the bottom plot shows sinusoidal control.

with $\omega=\sqrt{\frac{K_{J}}{J}}$. It can be verified by taking derivatives of $(10)$ :

$$
\begin{aligned}
\dot{q}(T) & =\underbrace{u(T)(1-\cos (0))}_{0}+\omega \int_{0}^{T} u(t) \sin (\omega(T-t)) \mathrm{d} t \\
\ddot{q}(T) & =\underbrace{u(T) \sin (0)}_{0}+\omega^{2} \int_{0}^{T} u(t) \cos (\omega(T-t)) \mathrm{d} t \\
& =\omega^{2}(\theta-q) .
\end{aligned}
$$

We assume $T$ to be fixed, i.e. the goal is to maximize the joint velocity at a known point in time. Then the integrand of (11) can be maximized for every $t$ independently by setting $u(t)=$ $u_{\max } \operatorname{sgn} \sin (\omega(T-t))$ leading to the overall maximum

$$
\begin{aligned}
\max _{u} \dot{q}(T) & =u_{\max } \omega \int_{0}^{T}|\sin (\omega(T-t))| \mathrm{d} t \\
& =u_{\max } \int_{0}^{\omega T}|\sin (x)| \mathrm{d} x \\
& =u_{\max }(2 n+1-\cos (\omega T-n \pi)),
\end{aligned}
$$

with $n=\left\lfloor\frac{\omega T}{\pi}\right\rfloor$. The last equation is obtained by splitting (15) at multiples of $\pi$ according to the sign of $\sin (x)$. The result is very promising (Fig. 19, top plot). Even for $\omega T=\pi$, i.e. half a cycle of the spring-mass eigenfrequency, the joint velocity can already be doubled. This is achieved by simply commanding maximum motor velocity, i.e. without any back and forth motion. For $\omega T=2 \pi$, i.e. a full cycle or going one times back and forth, the joint velocity can be quadrupled. Using more than a full cycle seems unrealistic for soccer as an application.

Of course the results refer to an idealized setting. In reality, the system would involve motor inertia, friction, damping, and torque limits. Damping and friction on the link side reduce the obtainable velocity but mainly when it is built up over many cycles, so they create no severe problem. Friction on the motor side only increases the torque needed, hence effectively reducing any torque limit. Motor inertia prohibits bang-bang control which would require infinite acceleration $\ddot{\theta}$. To analyze these effects, we now evaluate rather conservative sinusoidal control

$$
\begin{aligned}
u(t) & =u_{\max } \sin (\omega(T-t)) . \\
\dot{q}(T) & =u_{\max } \omega \int_{0}^{T} \sin ^{2}(\omega(T-t)) \mathrm{d} t \\
& =u_{\max }\left(\frac{\omega T}{2}-\frac{\sin (2 \omega T)}{4}\right) .
\end{aligned}
$$

As Fig. 19 (bottom plot) shows, the speedup reduces from 2 and 4 to $\frac{\pi}{2}$ and $\pi$ respectively. 
Torque limits do have an important effect that can be seen from the energy balance. A motor with limited velocity and torque can only generate limited power and hence energy can only built up $\propto T$ and velocity only $\propto \sqrt{T}$. As both control policies discussed above result in a linear built up of velocity they will at some point exceed the motor's torque limit.

When comparing these theoretical results in Fig. 19 to the practical ones in Fig. 17, some caution is needed. The experiments there show a back-and-forth motion, roughly corresponding to $\frac{\omega T}{\pi}=2$. So a factor of 4 could be achieved with an ideal velocity source, or $\pi \approx 3.14$ with sinusoidal control. In the experiments, only a factor of 2.45 has been achieved. However, $\dot{\theta}$ in Fig. 17 is far from being sinusoidal, let alone from an ideal step trajectory. Further, the VS-Joint has a progressive spring. So, from our view, the experiments correspond to the theory to the rough extend we expected from the simple model (6)-(8).

Another problem arising from the elasticity can be seen in (16) near $T=0$ (Fig. 19). The term $1-\cos (\omega T)$ has 0 derivative there, so in little time almost no velocity can be obtained. This is the usual problem that elasticity in the joints reduces joint dynamics. Overall, there are some problems in using elasticity to increase velocity. However, for sport robotics, the obtainable gains in our opinion far outweigh these problems.

\section{Conclusion}

In this paper, we analyzed safety and performance challenges imposed by the RoboCup 2050 vision of a human-robot soccer match. A key understanding we gained is the necessity of a new actuation paradigm, including elasticity (i.e. mechanical compliance) in the robot joints. This contributes to three important challenges of human-robot soccer:

Safety of the human. Joint elasticity decouples motor and link inertia. Hence, someone hit by the robot feels only the impact of the link at first. The impact of the motor inertia via the link side contact is delayed and less severe than if both happened at once. It can be further reduced by a collision detection mechanism. For this strategy to be effective, singularities must be avoided.

Protection of the robot. The same effect also protects the robot during an impact, because it gives the motor more time to decelerate. This reduces the peak gear torque, avoiding gear damage. Figuratively speaking, if a stiff robot bangs its fist on a table, it could hurt its shoulder. Joint elasticity prevents this. The benefit for the robot is therefore even higher than for the human!

Robot performance. Elasticity can store and release energy. Thereby it allows us to increase the joint velocity to a multiple of the maximum motor velocity. This makes motion control, in particular walking, much more difficult, but helps to close the gap in joint speed performance between humans and robots.

Of course, motion control, in particular walking and running with elastic joints is difficult. However, overall, joint elasticity has such a high potential for a humanoid soccer robot that these challenges should definitely be investigated in the near future.

Videos showing some simulation and the experiments presented throughout this paper can be downloaded from www. robotic.dlr.de/competitiveRobotics/.

\section{Acknowledgments}

We would especially like to thank Christian Ott and Markus Grebenstein for the fruitful discussions, leading to very interesting formulations of problems and deeper insight. Furthermore, we would like to thank Mirko Frommberger, Johann Heindl, Tim Bodenmüller, and Nadine Krüger for their great help. Exceptional thanks go to Martin Görner and Christoph Brachmann for the illustrations. Last but not least we would like to thank David and Mathias Nickl for their help and kindness.
Table 2

Mathematical symbols.

\begin{tabular}{ll}
\hline$\dot{\mathbf{x}}_{B, F, R} \in \mathfrak{R}^{3}$ & Velocity vector of ball, human foot, and robot foot \\
$m_{B}, F$ & Effective mass of the ball and foot \\
$e$ & Coefficient of restitution (COR) \\
$\tau_{J}$ & Joint torque \\
$\tau_{\mathrm{msr}}$ & Measured joint torque \\
$\phi_{1,2}$ & Angle of the first and second (foot-limb) axis \\
$h_{B}$ & Height of the ball before kick \\
$S_{l, w, f}$ & Coordinate system of limb, world, and foot \\
$\mathbf{n}_{C} \in \mathfrak{R}^{3}$ & Contact normal between foot and ball \\
$\varphi$ & Joint deflection \\
$q$ & Link position \\
$\theta$ & Motor position \\
$F_{\text {ext }}$ & Contact force \\
$F_{J}$ & Joint force \\
$x_{\text {kick }}$ & Kicking distance of the ball \\
$h_{\text {kick }}$ & Highest position of the ball after lob kick \\
$K_{J}$ & Joint stiffness \\
$J$ & Link inertia \\
$u$ & Control input/command \\
$\omega$ & Eigenfrequency \\
$E_{\text {Cov }}$ & Elasticity modulus of the compliant covering \\
$d_{\text {Cov }}$ & Thickness of the compliant covering \\
\hline
\end{tabular}

\section{References}

[1] H. Kitano, M. Asada, RoboCup humanoid challenge: That's one small step for a robot, one giant leap for mankind, in: IEEE/RSJ Int. Conf. on Intelligent Robots and Systems, IROS1998, Victoria, 1998, pp. 419-424.

[2] A. Lees, L. Nolan, The biomechanics of soccer: A review, Journal of Sport Sciences 16 (3) (1998) 211-234.

[3] Fédération Internationale de Football Association, Laws of the game 2006, 2006.

[4] O. Khatib, Inertial properties in robotic manipulation: An object-level framework, International Journal of Robotics Research 14 (1) (1995) 19-36.

[5] S. Haddadin, A. Albu-Schäffer, G. Hirzinger, Safety evaluation of physical human-robot interaction via crash-testing, in: Robotics: Science and Systems Conf., RSS2007, 2007, pp. 217-224.

[6] PHRIENDS: Physical human-robot interaction: Dependability and safety, PHRIENDS web site, 2007. http://www.phriends.eu/.

[7] SMErobot: The European Robot Initiative for Strengthening the Competitiveness of SMEs in Manufacturing, SMErobot web site, 2007. http://www. smerobot.org/.

[8] C. Ott, O. Eiberger, W. Friedl, B. Bäuml, U. Hillenbrand, C. Borst, A. AlbuSchäffer, B. Brunner, H. Hirschmüller, S. Kielhöfer, R. Konietschke, M. Suppa, T. Wimböck, F. Zacharias, G. Hirzinger, A humanoid two-arm system for dexterous manipulation, in: IEEE-RAS Int. Conf. on Humanoid Robots, HUMANOIDS2006, Genova, Italy, 2006, pp. 276-283.

[9] G. Hirzinger, N. Sporer, A. Albu-Schäffer, R. Krenn, A. Pascucci, M. Schedl, DLR's torque-controlled light weight robot III - are we reaching the technological limits now? in: IEEE Int. Conf. on Robotics and Automation, ICRA2002, Washington, DC, USA, 2002, pp. 1710-1716.

[10] G. Cheng, S.-H. Hyon, J. Morimoto, A. Ude, J.G. Hale, G. Colvin, W. Scroggin, S.C. Jacobsen, CB: A humanoid research platform for exploring neuroscience, Advanced Robotics 21 (10) (2007) 1097-1114.

[11] H. Kawasaki, T. Komatsu, K. Uchiyama, Dexterous anthropomorphic robot hand with distributed tactile sensor: Gifu hand II, IEEE/ASME Transactions Mechatronics 7 (2002) 296-303.

[12] W.T. Townsend, J.K. Salisbury, Mechanical design for whole-arm manipulation, in: P. Dario, G. Sandini, P. Aebischer (Eds.), Robots and Biological Systems: Towards a New Bionics? Springer, 1993, pp. 153-164.

[13] N. Hogan, Impedance control: An approach to manipulation: Part I - theory, Part II - implementation, Part III - applications, Journal of Dynamic Systems, Measurement and Control 107 (1985) 1-24.

[14] J. Craig, M. Raibert, A systematic method for hybrid position/force control of a manipulator, in: IEEE Computer Software Applications Conf., 1979, pp. $446-451$.

[15] A. Albu-Schäffer, S. Haddadin, Ch. Ott, A. Stemmer, T. Wimböck, G. Hirzinger, The DLR lightweight robot - lightweight design and soft robotics control concepts for robots in human environments, Industrial Robot Journal 34 (5) (2007) 376-385.

[16] G. Tonietti, R. Schiavi, A. Bicchi, Design and control of a variable stiffness actuator for safe and fast physical human/robot interaction, in: Int. Conf. on Robotics and Automation, ICRA2005, Barcelona, Spain, 2005, pp. 528-533. 
[17] B. Vanderborght, B. Verrelst, R. Van Ham, M. Van Damme, D. Lefeber, B.M.Y. Duran, P. Beyl, Exploiting natural dynamics to reduce energy consumption by controlling the compliance of soft actuators, International Journal of Robotics Research 25 (4) (2006) 343-358.

18] T. Morita, H. Iwata, S. Sugano, Development of human symbiotic robot: WENDY, in: IEEE Int. Conf. on Robotics and Automation, ICRA1999, Detroit, USA, 1999, pp. 3183-3188.

[19] A. Bicchi, G. Tonietti, Fast and soft arm tactics: Dealing with the safetyperformance trade-off in robot arms design and control, IEEE Robotics and Automation Magazine 11 (2004) 22-33.

[20] S.A. Migliore, E.A. Brown, S.P. DeWeerth, Biologically inspired joint stiffness control, in: IEEE Int. Conf. on Robotics and Automation, ICRA2005, Barcelona, Spain, 2005.

[21] G. Palli, C. Melchiorri, T. Wimboeck, M. Grebenstein, G. Hirzinger, Feedback linearization and simultaneous stiffness-position control of robots with antagonistic actuated joints, in: IEEE Int. Conf. on Robotics and Automation, ICRA2007, Rome, Italy, 2007, pp. 2928-2933.

[22] S. Wolf, G. Hirzinger, A new variable stiffness design: Matching requirements of the next robot generation, in: IEEE Int. Conf. on Robotics and Automation, ICRA 2008, Pasadena, USA, 2008, pp. 1741-1746.

[23] K. Nishiwaki, T. Sugihara, S. Kagami, F. Kanehiro, M. Inaba, H. Inoue, Design and development of research platform for perceptionaction integration in humanoid robot: H6, in: IEEE/RSJ Int. Conf. on Intelligent Robots and Systems, IROS2000, Takamatsu, Japan, 2000, pp. 1559-1564.

[24] K. Hirai, M. Hirose, Y. Haikawa, T. Takenaka, The development of hond humanoid robot, in: IEEE Int. Conf. on Robotics and Automation, ICRA1998, Leuven, Belgium, 1998, pp. 1321-1326.

[25] M. Hirose, Y. Haikawa, T. Takenaka, K. Hirai, Development of humanoid robot ASIMO, in: IEEE/RSJ Int. Conf. on Intelligent Robots and Systems, IROS2001: Workshop 2, Maui, USA, 2001.

[26] S. Lohmeier, T. Buschmann, H. Ulbrich, F. Pfeiffer, Modular joint design for a performance enhanced humanoid robot, in: IEEE Int. Conf. on Robotics and Automation, ICRA2006, Orlando, USA, 2006, pp. 88-93.

[27] Y. Ogura, H. Aikawa, K. Shimomura, H. Kondo, A. Morishima, Hun ok Lim, A. Takanishi, Development of a new humanoid robot wabian-2, in: IEEE Int Conf. on Robotics and Automation, ICRA2006, Orlando, USA, 2006, pp. 76-81.

[28] J.-Y. Kim, I.-W. Park, J. Lee, M.S. Kim, B.K. Cho, J.-H. Oh, System design and dynamic walking of humanoid robot KHR-2, in: Int. Conf. on Robotics and Automation, ICRA2005, Barcelona, Spain, 2005, pp. 1443-1448.

[29] K. Kaneko, F. Kanehiro, S. Kajita, H. Hirukawa, T. Kawasaki, M. Hirata, K. Akachi, T. Isozumi, Humanoid Robot HRP-2, in: Int. Conf. on Robotics and Automation, ICRA2004, New Orleans, USA, 2004, pp. 1083-1090.

[30] S. Shirata, A. Konno, M. Uchiyama, Design and development of a light-weight biped humanoid robot Saika-4, in: IEEE/RSJ Int. Conf. on Intelligent Robots and Systems, IROS2004, Sendai, Japan, 2004, pp. 148-153.

31] J. Yamaguchi, S. Inoue, D. Nishino, A. Takanishi, Development of a bipeda humanoid robot having antagonistic driven joints and three DOF trunk, in: IEEE/RSJ Int. Conf. on Intelligent Robots and Systems, IROS1998, Victoria, B.C. Canada, 1998, pp. 96-101.

[32] J. Yamaguchi, D. Nishino, A. Takanishi, Realization of dynamic biped walking varying joint stiffness using antagonistic driven joints, in: IEEE Int. Conf. on Robotics and Automation, ICRA1998, Leuven, Belgium, 1998, pp. 2022-2029.

33] D. Hobbelen, T de Boer, M. Wisse, System overview of bipedal robots flame and tulip: Tailor-made for limit cycle walking, in: IEEE/RSJ Int. Conf. on Intelligent Robots and Systems, IROS2008, Nice, France, 2008, pp. 2486-2491.

[34] S. Kajita, K. Kaneko, M. Morisawa, S. Nakaoka, H. Hirukawa, ZMP-based biped running enhanced by toe springs, in: Int. Conf. on Robotics and Automation, ICRA2007, Rome, Italy, 2007, pp. 3363-3369.

[35] B.M. Nigg, B.R. MacIntosh, J. Mester (Eds.), Biomechanics and Biology of Movement, Human Kinetics Pub Inc., Champaign, IL, USA, 2000.

[36] Issac Asimov, The Caves Of Steel, A Robot Novel, Doubleday, Garden City, New York, 1954.

[37] M. Zinn, O. Khatib, B. Roth, A new actuation approach for human friendly robot design, The International Journal of Robotics Research 23 (2004) 379-398.

[38] S. Haddadin, A. Albu-Schäffer, G. Hirzinger, The role of the robot mass and velocity in physical human-robot interaction - Part I: Unconstrained blun impacts, in: IEEE Int. Conf. on Robotics and Automation, ICRA2008, Pasadena, USA, 2008, pp. 1331-1338.

[39] S. Haddadin, A. Albu-Schäffer, G. Hirzinger, The role of the robot mass and velocity in physical human-robot interaction - Part II: Constrained blun impacts, in: IEEE Int. Conf. on Robotics and Automation, ICRA2008, Pasadena, USA, 2008, pp. 1339-1345.

[40] M. Grebenstein, P. van der Smagt, Antagonism for a highly anthropomorphic hand-arm system, Advanced Robotics 22 (1) (2008) 39-55.

[41] Alin Albu-Schäffer, Oliver Eiberger, Markus Grebenstein, Sami Haddadin, Christian Ott, Thomas Wimböck, Sebastian Wolf, Gerd Hirzinger, Soft robotics, in: Adaptable Compliance / Variable Stiffness for Robotic Applications, Robotics \& Automation Magazine 15 (3) (2008) 20-30 (special issue)

[42] R.F. Chandler, C.E. Clauser, J.T. McConville, H.M. Reynolds, J.W. Young, Investigation of inertial properties of the human body, Technical Report DOT HS-801 430, Aerospace Medical Research Laboratory, 1975.
[43] S. Haddadin, T. Laue, U. Frese, G. Hirzinger, Foul 2050: Thoughts on physical interaction in human-robot soccer, in: IEEE/RSJ Int. Conf. on Intelligent Robots and Systems, IROS2007, San Diego, USA, 2007, pp. 3243-3250.

[44] H. Nunome, T. Asai, Y. Ikegamiand, S. Sakurai, Three-dimensional kinetic analysis of side-foot and instep soccer kicks, Medicine \& Science in Sports \& Exercise 34 (12) (2002) 2028-2036.

[45] P. Kulvanit, O. von Stryk, Robocup soccer humanoid league rules and setup for the 2008 competition in Suzhou, China, 2008.

[46] A. McGrath, J. Ozanne-Smith, Heading injuries out of soccer: A review of the literature, Technical Report 125, Monash University Accident Research Center, 1997.

[47] J.A. Newman, N. Shewchenko, E. Welbourne, A proposed new biomechanica head injury assessment function - the maximum power index, SAE paper 2000-01-SC16 Stapp Car Crash Journal 44 (2000) 215-247.

[48] N. Shewchenko, N. Withnall, M. Keown, R. Gittens, J. Dvorak, Heading in football. Part 1: Development of biomechanical methods to investigate head response, British Journal Sports Medicine 39 (2005) 10-25.

[49] H.-D. Burkhard, D. Duhaut, M. Fujita, P. Lima, R. Murphy, R. Rojas, The road to RoboCup 2050, IEEE Robotics and Automation Magazine 9 (2) (2002) 31-38.

[50] American Association for Automotive Medicine, The abbreviated injury scale, 1980.

[51] H. Johannsen, V. Schindler, Review of the abdomen injury criteria, Technical Report AP-SP51-0039-B, Institut National de Reserche sur les Transports et leur Sécurité, 2005.

[52] J.M. Cavanaugh, G.W. Nyquist, S.J. Goldberg, A.I. King, Lower abdominal impact tolerance and response, SAE Paper No. 861878, in: Proc. 30th Stapp Car Crash Conf., 1986.

[53] T.E. Andersen, Á. Árnason, L. Engebretsen, R. Bahr, Mechanisms of head injuries in elite football, British Journal Sports Medicine 38 (2004) 690-696.

[54] Douglas L. Allsop, Charles Y. Warner, Milton G. Wille, Dennis C. Schneider, Alan M. Nahum, Facial impact response - A comparison of the Hybrid III dummy and human cadaver, SAE Paper No.881719, in: Proc. 32th Stapp Car Crash Conf. 1988, pp. 781-797.

[55] J. Melvin, Human tolerance to impact conditions as related to motor vehicle design, in: SAE J885 APR80, 1980.

[56] European Commission Framework, Improved frontal impact protection through a world frontal impact dummy, Project No. GRD1 1999-10559, 2003.

[57] D. Kallieris, A. Rizzetti, R. Mattern, S. Jost, P. Priemer, M. Unger, Response and vulnerability of the upper arm through side air bag deployment, SAE Transactions 120 (2004) 143-152.

[58] C. Withnall, N. Shewchenko, R. Gittens, J. Dvorak, Biomechanical investigation of head impacts in football, British Journal of Sports Medicine 39 (2005) 49-57.

59] I.P. Herman, Physics of the Human Body, Springer Verlag, 2007.

[60] A. De Luca, A. Albu-Schäffer, S. Haddadin, G. Hirzinger, Collision detection and safe reaction with the DLR-III lightweight manipulator arm, in: IEEE/RSJ Int. Conf. on Intelligent Robots and Systems 2006, IROS2006, Beijing, China, 2006 pp. $1623-1630$.

[61] S. Haddadin, Evaluation criteria and control structures for safe human-robot interaction, Master's Thesis, Technical University of Munich (TUM) \& German Aerospace Center (DLR), 122005.

[62] G.A. Pratt, M. Williamson, Series elastics actuators, in: IEEE/RS] Int. Conf. on Intelligent Robots and Systems 1995, IROS1995, Victoria, Canada, 1995, pp. 399-406.

[63] S. Haddadin, A. Albu-Schäffer, A. De Luca, G. Hirzinger, Collision detection \& reaction: A contribution to safe physical human-robot interaction, in: IEEE/RS Int. Conf. on Intelligent Robots and Systems, IROS2008, Nice, France, 2008 pp. 3356-3363.

[64] H. Schempf, C. Kraeuter, M. Blackwell, ROBOLEG: A robotic soccer-ball kicking leg, in: IEEE Int. Conf. on Robotics and Automation, ICRA1995, Nagoya, Aichi, Japan, vol. 2, 1995, pp. 1314-1318

[65] D. Paluska, H. Herr, The effect of series elasticity on actuator power and work output: Implications for robotic and prosthetic joint design, Robotics and Autonomous Systems 54 (2006) 667-673.

[66] M. Okada, S. Ban, Y. Nakamura, Skill of compliance with controlled charging/discharging of kinetic energy, in: IEEE Int. Conf. on Robotics and Automation, ICRA2002, Washington, USA, 2002, pp. 2455-2460.

[67] S. Behnke, M. Schreiber, J. Stückler, H. Schulz, M. Böhnert, K. Meier, NimbRo teensize 2008 team description, in: RoboCup 2008: Robot Soccer World Cup XII Preproceedings, 2008.

[68] M. Spong, Modeling and control of elastic joint robots, IEEE Journal of Robotics and Automation (1987) 291-300.

[69] Sami Haddadin, Alin Albu-Schäffer, Gerd Hirzinger, Approaching Asimov's 1st Law, in: C. Bartneck, T. Kanada (Eds.), HRI Caught on Film, Proceedings of the 2nd ACM/IEEE International Conference on Human-Robot Interaction, Washington DC, USA, 2007, pp. 177-184.

[70] Dongjun Shin, Irene Sardellitti, Oussama Khatib, Hybrid actuation approach for human-friendly robot design, in: IEEE Int. Conf. on Robotics and Automation (ICRA 2008), Pasadena, USA, 2008, pp. 1741-1746.

[71] A. Bicchi, M. Bavaro, G. Boccadamo, D. De Carli, R. Filippini, G. Grioli, M. Piccigallo, A. Rosi, R. Schiavi, S. Sen, G. Tonietti, Physical humanrobot interaction: Dependability, safety, and performance, in: Int. Workshop Advanced Motion Control, 2008, pp.9-14. 


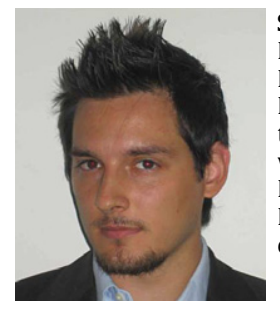

Sami Haddadin received his Dipl.-Ing. degree in Electrical Engineering in 2006 from the Technical University of Munich and holds an Honours degree in Technology Management from the Technical University of Munich and the Ludwig Maximilian University Munich. He currently works at the German Aerospace Center (DLR) as a Research Engineer. His main research topics are physical Human-Robot Interaction, robot control, and safety and dependability in robotics.

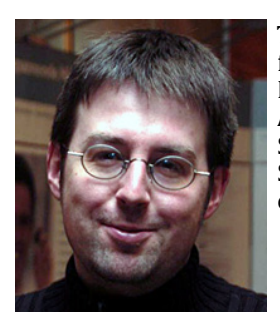

Tim Laue received his diploma in computer science from the University of Bremen, Germany, in 2004. $\mathrm{He}$ is currently with the German Research Center for Artificial Intelligence (DFKI) at the research group for Safe and Secure Cognitive Systems in Bremen, Germany. Since 2002, he continuously participates in RoboCup competitions within different robot soccer leagues.

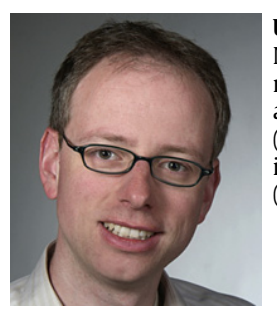

Udo Frese received his Ph.D. from University of ErlangenNürnberg in 2004. He is now assistant professor for real-time computer vision at University of Bremen and affiliated with the German Center for Artificial Intelligence (DFKI). His research topics are real-time computer vision, in particular simultaneous localization and mapping (SLAM) and computer vision for sport robotics.

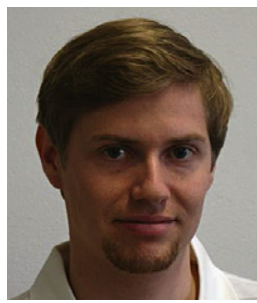

Sebastian Wolf received his Dipl.-Ing. degree in Mechanical Engineering 2004 from the Technical University of Munich, Germany. He joined the Institute for Feingerätebau und Mikrotechnik at the Technical University of Munich, Germany as a Research Associate in 2004. Since 2005 he is a Research Engineer at the Institute of Robotics and Mechatronics at German Aerospace Center (DLR). His main research interest is in variable stiffness actuation.

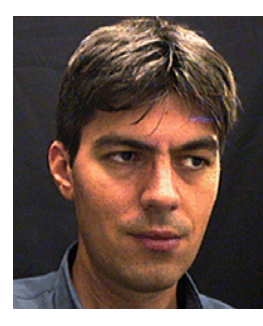

Alin Albu-Schäffer received his Dipl.-Ing.degree in Electrical Engineering from the Technical University of Timisoara, Romania in 1993 and the Ph.D. degree in Control Systems in 2002 from the Technical University of Munich, Germany. Since 1995, he has been with the Institute of Robotics and Mechatronics at the German Aerospace Center (DLR), where he is currently coordinating control and advanced manipulation activities. His research interests include robot modelling and control, nonlinear control, flexible joint robots, impedance and force control, physical human-robot interaction.

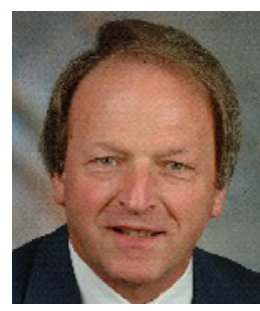

Gerd Hirzinger received his Dipl.-Ing. degree and his Ph.D. degree from the Technical University of Munich, in 1969 and 1974 respectively. In 1991 he received a joint professorship from the Technical University of Munich. Since 1992 he has been director at DLR's institute for "robotics and mechatronics". He received several national and international awards, e.g. in 1994 the Joseph-Engelberger-Award for achievements in the robotic science and in 1995 the Leibniz-Award, the highest scientific award in Germany and the JARA (Japan robotics association) Award. In 2005 he received the IEEE Pioneer Award of the Robotics and Automation Society and the "honorary citizenship" of Budapest Tech, and in 2007 the IEEE Field Award "Robotics and Automation". 